\title{
Equations of Electrodynamics
}

\author{
Pavel Gorev \\ JSC "Polet" \\ Leading Researcher \\ Candidate of Technical Sciences
}
Nizhny Novgorod
Russia

E-mail: pppay7733@yahoo.com

\begin{abstract}
Maxwell's equations are valid only for a stationary observation point, therefore, to adequately describe real processes so far we have had to move to a moving reference frame. This paper presents the equations of electrodynamics for the moving observation point, it is shown that plane and spherical electromagnetic waves are their solutions, while the spherical wave propagates only outward, which cannot be said about Maxwell's equations. The fields of uniformly moving charges are also solutions of the equations. Now there is no need to move to a moving reference frame, to use four-dimensional space and covariant form of equations. The question of finding a universal form of the equations that allows a solution in the form of the field of an arbitrarily moving charge remains open. This raises the question of the existence of a two-parameter group of transformations of electromagnetic fields along with the known oneparameter group has been posed. The phenomena derived from the equations, which make an additional contribution to the phase overrun in the Aharonov-Bohm effect are considered. The equation of motion of a charged particle in an electromagnetic field without simplifying approximations is considered, which allows us to take into account the radiation effects. It is shown that the fields in a moving observation point depend on its velocity and acceleration. In particular, although in a constant uniform electric field a force $q \mathbf{E}$ acts on a motionless charged particle, but on the same motionless but not fixed particle the force $4 / 3 q \mathbf{E}$ acts already, because it has a nonzero acceleration and the electric field at this point is larger. As the speed increases, the field decreases, and when it reaches the speed of light, when the particle stops accelerating, the force again becomes equal to $q \mathbf{E}$. The principle of operation of an unconventional alternator in a constant electric field and its corresponding engine, as well as new types of direct and impulse current generators, predicted by the equations, are described.
\end{abstract}

Key words: electrodynamics, plane electromagnetic wave, spherical electromagnetic wave, group of transformations, Aharonov-Bohm effect, equation of motion, alternator, impulse current generator 


\section{Introduction}

The laws of electrodynamics, formulated by Maxwell, allowed him to predict the existence of electromagnetic waves. But this presupposition was based not on Maxwell's equations themselves, but on the wave equations constructed on their basis, the set of solution of which need not coincide with the set of solutions of the original equations. For example, a spherical wave is a solution of wave equations, but not of Maxwell's equations, besides, this solution allows propagation both outward and inward. We would like to find equations that describe electrodynamics more correctly. Such an attempt is made in this paper.

\section{Equations of Electrodynamics}

Further, the terms and designations from the book [1] are used. In [1] (Chapter 1, Paragraph 4, formula (1.7)), a law for the circulation of the electric field strength vector $\mathbf{E}$ is formulated:

$$
\oint_{L} \mathbf{E} \cdot d \mathbf{l}=-\frac{d}{d t} \int_{S} \mathbf{B} \cdot d \mathbf{s}
$$

Here $L$ is an arbitrary closed contour, $S$ is an arbitrary surface bounded by the contour $L, \mathbf{B}$ is the magnetic induction vector, $\mathbf{n}$ is the normal vector to the surface $S$ at some point, $d \mathbf{s}=\mathbf{n} d s$, $(\cdot)$ is the scalar product icon.

If the points of the contour have a nonzero velocity, then equation (1) looks as follows:

$$
\oint_{L} \mathbf{E} \cdot d \mathbf{l}=-\int_{S} \frac{\partial \mathbf{B}}{\partial t} \cdot d \mathbf{s}+\oint_{L}(\mathbf{v} \times \mathbf{B}) \cdot d \mathbf{l}
$$

where $\mathbf{v}$ is the velocity of the contour point in the selected frame of reference, $(\times)$ is the vector product icon. Similarly, for a magnetic field, we can write

$$
c^{2} \oint_{L} \mathbf{B} \cdot d \mathbf{l}=\int_{S} \frac{\partial \mathbf{E}}{\partial t} \cdot d \mathbf{s}+\frac{I}{\varepsilon_{0}}-\oint_{L}(\mathbf{v} \times \mathbf{E}) \cdot d \mathbf{l}
$$

Here $c$ is the speed of light, $\varepsilon_{0}$ is the electric constant, $I$ is the total electric current through the surface $S$.

The physical manifestations of the second term in equation (2) are found in the presence of a constant magnetic field, when the derivative $\mathbf{B}$ is equal to zero, but the circulation of the vector $\mathbf{E}$ is nonzero. For example, if we consider the rotation of the wire frame in a constant magnetic field, as happens, for example, in an alternator, then in (2) the first term on the right side is zero, and the electromotive force (EMF) is generated only due to the second term. The resulting from the integration of the second term in (2) along the contour of the EMF for any shape of the frame coincides with the EMF, which can be calculated from the rate of change of the magnetic flux through the surface area of the frame.

This coincidence ensures the correct formulation of the Faraday law of electromagnetic induction for both an alternating field and a constant field, as well as any combination of them.

This is true for an alternator with fixed magnets and a rotating frame. If the frame is stationary and the magnets rotate, then on the contrary, the second term is zero due to the 
absence of velocity in the frame elements, but the magnetic field on the surface, stretched on the frame, changes direction and its time derivative becomes non-zero. The integral of its normal to the surface component provides the EMF, the value of which again coincides with the EMF calculated by Faraday's law of electromagnetic induction. In Tilley's scheme, however, both terms in the right-hand side of (2) are zero, so there is no induction.

Applying Stokes' theorem to integrals along a contour, we obtain

$$
\begin{aligned}
& \int_{S} \operatorname{rot} \mathbf{E} \cdot d \mathbf{s}=-\int_{S} \frac{\partial \mathbf{B}}{\partial t} \cdot d \mathbf{s}+\int_{S} \operatorname{rot}(\mathbf{v} \times \mathbf{B}) \cdot d \mathbf{s} \\
& c^{2} \int_{S} \operatorname{rot} \mathbf{B} \cdot d \mathbf{s}=\int_{S} \frac{\partial \mathbf{E}}{\partial t} \cdot d \mathbf{s}-\int_{S} \operatorname{rot}(\mathbf{v} \times \mathbf{E}) \cdot d \mathbf{s}+\int_{S} \frac{\hat{\mathbf{j}}}{\varepsilon_{0}} \cdot d \mathbf{s}
\end{aligned}
$$

where $\hat{\mathbf{j}}$ is the current density at a point of the surface $S$. We assume that the Stokes' theorem is applicable to a moving frame and, accordingly, a moving surface.

Before proceeding with further consideration, we eliminate some asymmetry of equations (4) and (5) by adding one more term to them on the right-hand side

$$
\begin{gathered}
\int_{S} \operatorname{rot} \mathbf{E} \cdot d \mathbf{s}=-\int_{S} \frac{\partial \mathbf{B}}{\partial t} \cdot d \mathbf{s}+\int_{S} \operatorname{rot}(\mathbf{v} \times \mathbf{B}) \cdot d \mathbf{s}-\frac{1}{c^{2}} \int_{S} \frac{\partial(\mathbf{v} \times \mathbf{E})}{\partial t} \cdot d \mathbf{s} \\
c^{2} \int_{S} \operatorname{rot} \mathbf{B} \cdot d \mathbf{s}=\int_{S} \frac{\partial \mathbf{E}}{\partial t} \cdot d \mathbf{s}-\int_{S} \operatorname{rot}(\mathbf{v} \times \mathbf{E}) \cdot d \mathbf{s}+\int_{S} \frac{\partial(\mathbf{v} \times \mathbf{B})}{\partial t} \cdot d \mathbf{s}+\int_{S} \frac{\hat{\mathbf{j}}}{\varepsilon_{0}} \cdot d \mathbf{s}
\end{gathered}
$$

Since the surface $S$ is arbitrary, we can write

$$
\begin{aligned}
\operatorname{rot} \mathbf{E} & =-\frac{\partial \mathbf{B}}{\partial t}+\operatorname{rot}(\mathbf{v} \times \mathbf{B})-\frac{1}{c^{2}} \frac{\partial(\mathbf{v} \times \mathbf{E})}{\partial t} \\
c^{2} \operatorname{rot} \mathbf{B} & =\frac{\partial \mathbf{E}}{\partial t}-\operatorname{rot}(\mathbf{v} \times \mathbf{E})-\frac{\partial(\mathbf{v} \times \mathbf{B})}{\partial t}+\frac{\hat{\mathbf{j}}}{\varepsilon_{0}}
\end{aligned}
$$

Let us write equations (8) and (9) for free space without currents and charges

$$
\begin{gathered}
\operatorname{rot} \mathbf{E}=-\frac{\partial \mathbf{B}}{\partial t}+\operatorname{rot}(\mathbf{v} \times \mathbf{B})-\frac{1}{c^{2}} \frac{\partial(\mathbf{v} \times \mathbf{E})}{\partial t} \\
c^{2} \operatorname{rot} \mathbf{B}=\frac{\partial \mathbf{E}}{\partial t}-\operatorname{rot}(\mathbf{v} \times \mathbf{E})-\frac{\partial(\mathbf{v} \times \mathbf{B})}{\partial t}
\end{gathered}
$$

Let us check whether the solution in the form of a plane electromagnetic wave propagating with a constant velocity $\mathbf{u}$ in the direction of the $x$-axis and having nonzero components $E_{y}$ and $B_{z}$. satisfies these equations. Let us pass from the vector $\mathbf{B}$ to the new vector $\mathbf{B}^{\prime}=\mathbf{c B}$, for it the equations take the form

$$
\begin{gathered}
\operatorname{rot} \mathbf{E}=-\frac{1}{\mathrm{c}} \frac{\partial \mathbf{B}^{\prime}}{\partial t}+\frac{1}{\mathrm{c}} \operatorname{rot}\left(\mathbf{v} \times \mathbf{B}^{\prime}\right)-\frac{1}{c^{2}} \frac{\partial(\mathbf{v} \times \mathbf{E})}{\partial t} \\
\operatorname{rot} \mathbf{B}^{\prime}=\frac{1}{\mathrm{c}} \frac{\partial \mathbf{E}}{\partial t}-\frac{1}{\mathrm{c}} \operatorname{rot}(\mathbf{v} \times \mathbf{E})-\frac{1}{c^{2}} \frac{\partial\left(\mathbf{v} \times \mathbf{B}^{\prime}\right)}{\partial t}
\end{gathered}
$$

For the vectors included as terms in the first equation, we find the component $z$, assuming that the vector parameter $\mathbf{v}$ has only the component $x$ that is different from zero. In the $\operatorname{rot} \mathbf{E}$ vector, only the component $z$, which is equal to $\frac{\partial E_{y}}{\partial x}$, in the vector $\left(\mathbf{v} \times \mathbf{B}^{\prime}\right)$, only the component $y$, equal to $-v_{x} B_{z}^{\prime}$, is not equal to zero, in the vector $\operatorname{rot}\left(\mathbf{v} \times \mathbf{B}^{\prime}\right)$, only the component $z$, equal to - 
$v_{x} \frac{\partial B_{z}^{\prime}}{\partial x}$, in the vector $(\mathbf{v} \times \mathbf{E})$, only the component $z$, equal to $v_{x} E_{y}$, is different from zero. After substituting these values, we obtain

$$
\frac{\partial E_{y}}{\partial x}=-\frac{1}{\mathrm{c}} \frac{\partial B_{z}^{\prime}}{\partial t}-\frac{v_{x}}{\mathrm{c}} \frac{\partial B_{z}^{\prime}}{\partial x}-\frac{v_{x}}{\mathrm{c}^{2}} \frac{\partial E_{y}}{\partial t}
$$

For the vectors included as terms in the second equation, we find the component $y$. The vector $\operatorname{rot} \mathbf{B}^{\prime}$ has only the component $y$, which is equal to $-\frac{\partial B_{z}^{\prime}}{\partial x}$ the vector $(\mathbf{v} \times \mathbf{E})$ has only the component $z$, equal to $v_{x} E_{y}$, different from zero, the vector $\operatorname{rot}(\boldsymbol{v} \times \mathbf{E})$ has only the component $y$, equal to $-v_{x} \frac{\partial E_{y}}{\partial x}$. The vector $\left(\mathbf{v} \times \mathbf{B}^{\prime}\right)$ has only the $y$ component equal to $-v_{x} B_{z}^{\prime}$. After substituting these values, we get

$$
-\frac{\partial B_{z}^{\prime}}{\partial x}=\frac{1}{\mathrm{c}} \frac{\partial E_{y}}{\partial t}+\frac{v_{x}}{\mathrm{c}} \frac{\partial E_{y}}{\partial x}+\frac{v_{x}}{\mathrm{c}^{2}} \frac{\partial B_{z}^{\prime}}{\partial t}
$$

Consider the possibility of a solution in the form of the following function

$$
E_{y}(x, t)=B_{z}^{\prime}(x, t)=f\left(x-u_{x} t\right)
$$

Substituting this solution, we get

$$
\begin{aligned}
& f^{\prime}=-\frac{1}{\mathrm{c}}\left(-u_{x} f^{\prime}\right)-\frac{v_{x}}{\mathrm{c}} f^{\prime}-\frac{v_{x}}{\mathrm{c}^{2}}\left(-u_{x} f^{\prime}\right) \\
& -f^{\prime}=\frac{1}{\mathrm{c}}\left(-u_{x} f^{\prime}\right)+\frac{v_{x}}{\mathrm{c}} f^{\prime}+\frac{v_{x}}{\mathrm{c}^{2}}\left(-u_{x} f^{\prime}\right)
\end{aligned}
$$

if we put the parameter of the equation equal to the propagation velocity $v_{x}=u_{x}$ and denote $\delta=\frac{u_{x}}{\mathrm{c}}$, then

$$
\begin{gathered}
1=\delta-\delta+\delta \delta \\
-1=-\delta+\delta-\delta \delta \\
\delta^{2}=1 \\
\delta= \pm 1 \\
u_{x}= \pm c
\end{gathered}
$$

Propagation of the field excitation is possible in two directions and only at the speed of light. Note that a similar consideration for Maxwell's equations leads to the solution $\delta=1$, that is, the wave can propagate only in one direction of the $x$-axis. The velocity $\mathbf{u}$ is the velocity of the wave, and the velocity $\mathbf{v}$ is a free parameter of the equation that can be adjusted for specific wave realizations. Now it is clear that it is necessary to include additional terms in equations (6) and (7), without them plane waves would not be solutions to the equations.

Let's check whether the solution in the form of a spherical electromagnetic wave propagating with a constant velocity $\mathbf{u}$ in the radial direction satisfies the equations of electrodynamics. Consider a ray from the origin with a direction along the $x$-axis and assume that the field has nonzero components only $E_{y}$ and $B_{z}$. Again for $\mathbf{B}^{\prime}=\mathbf{c B}$, the equations have the form

Again we get

$$
\begin{aligned}
& \operatorname{rot} \mathbf{E}=-\frac{1}{\mathrm{c}} \frac{\partial \mathbf{B}^{\prime}}{\partial t}+\frac{1}{\mathrm{c}} \operatorname{rot}\left(\mathbf{v} \times \mathbf{B}^{\prime}\right)-\frac{1}{c^{2}} \frac{\partial(\mathbf{v} \times \mathbf{E})}{\partial t} \\
& \operatorname{rot} \mathbf{B}^{\prime}=\frac{1}{\mathrm{c}} \frac{\partial \mathbf{E}}{\partial t}-\frac{1}{\mathrm{c}} \operatorname{rot}(\mathbf{v} \times \mathbf{E})-\frac{1}{c^{2}} \frac{\partial\left(\mathbf{v} \times \mathbf{B}^{\prime}\right)}{\partial t}
\end{aligned}
$$

$$
\frac{\partial E_{y}}{\partial x}=-\frac{1}{\mathrm{c}} \frac{\partial B_{z}^{\prime}}{\partial t}-\frac{v_{x}}{\mathrm{c}} \frac{\partial B_{z}^{\prime}}{\partial x}-\frac{v_{x}}{\mathrm{c}^{2}} \frac{\partial E_{y}}{\partial t}
$$




$$
-\frac{\partial B_{z}^{\prime}}{\partial x}=\frac{1}{\mathrm{c}} \frac{\partial E_{y}}{\partial t}+\frac{v_{x}}{\mathrm{c}} \frac{\partial E_{y}}{\partial x}+\frac{v_{x}}{\mathrm{c}^{2}} \frac{\partial B_{z}^{\prime}}{\partial t}
$$

Consider the possibility of a solution in the form of the following function

$$
E_{y}(x, t)=B_{z}^{\prime}(x, t)=\frac{f\left(r-u_{x} t\right)}{r}
$$

where $r=\sqrt{x^{2}+y^{2}+z^{2}}$,

$$
\begin{aligned}
& \frac{\partial}{\partial x}\left(\frac{f\left(r-u_{x} t\right)}{r}\right)= \frac{\partial}{\partial r}\left(\frac{f\left(r-u_{x} t\right)}{r}\right) \frac{\partial r}{\partial x}=\left(\frac{f^{\prime}\left(r-u_{x} t\right)}{r}+\left(-\frac{f\left(r-u_{x} t\right)}{r^{2}}\right)\right)\left(\frac{x}{r}\right) \\
& \frac{\partial}{\partial t}\left(\frac{f\left(r-u_{x} t\right)}{r}\right)=\left(\frac{1}{r}\right) \frac{\partial f\left(r-u_{x} t\right)}{\partial t}=\left(\frac{f^{\prime}\left(r-u_{x} t\right)}{r}\right)\left(-u_{x}\right) \\
& \frac{\partial}{\partial x}\left(\frac{f\left(r-u_{x} t\right)}{r}\right)=\frac{\partial}{\partial x}\left(f\left(r-u_{x} t\right)\right) \frac{1}{r}+f\left(r-u_{x} t\right) \frac{\partial}{\partial x}\left(\frac{1}{r}\right) \\
&=f^{\prime}\left(r-u_{x} t\right) \frac{\partial r}{\partial x} \frac{1}{r}+f\left(r-u_{x} t\right)\left(-\frac{1}{r^{2}} \frac{\partial r}{\partial x}\right) \\
&=\frac{f^{\prime}\left(r-u_{x} t\right)}{r}\left(\frac{x}{r}\right)-\frac{f\left(r-u_{x} t\right)}{r^{2}}\left(\frac{x}{r}\right)=\frac{f^{\prime}}{r}\left(\frac{x}{r}\right)-\frac{f}{r^{2}}\left(\frac{x}{r}\right)
\end{aligned}
$$

Substituting this solution, we get

$$
\begin{aligned}
& \left(\frac{x}{r}\right)\left(\frac{f^{\prime}}{r}-\frac{f}{r^{2}}\right)=-\frac{1}{\mathrm{c}}\left(\left(\frac{f^{\prime}}{r}\right)\left(-u_{x}\right)\right)-\frac{v_{x}}{\mathrm{c}}\left(\frac{x}{r}\right)\left(\frac{f^{\prime}}{r}-\frac{f}{r^{2}}\right)-\frac{v_{x}}{\mathrm{c}^{2}}\left(\left(\frac{f^{\prime}}{r}\right)\left(-u_{x}\right)\right) \\
& -\left(\frac{x}{r}\right)\left(\frac{f^{\prime}}{r}-\frac{f}{r^{2}}\right)=\frac{1}{\mathrm{c}}\left(\left(\frac{f^{\prime}}{r}\right)\left(-u_{x}\right)\right)+\frac{v_{x}}{\mathrm{c}}\left(\frac{x}{r}\right)\left(\frac{f^{\prime}}{r}-\frac{f}{r^{2}}\right)+\frac{v_{x}}{\mathrm{c}^{2}}\left(\left(\frac{f^{\prime}}{r}\right)\left(-u_{x}\right)\right)
\end{aligned}
$$

If we select a point on the $x$-axis in the positive direction from the origin, then $x=r$ and we can write

$$
\begin{aligned}
& \left(\frac{f^{\prime}}{r}-\frac{f}{r^{2}}\right)=-\frac{1}{\mathrm{c}}\left(\left(\frac{f^{\prime}}{r}\right)\left(-u_{x}\right)\right)-\frac{v_{x}}{\mathrm{c}}\left(\frac{f^{\prime}}{r}-\frac{f}{r^{2}}\right)-\frac{v_{x}}{\mathrm{c}^{2}}\left(\left(\frac{f^{\prime}}{r}\right)\left(-u_{x}\right)\right) \\
& -\left(\frac{f^{\prime}}{r}-\frac{f}{r^{2}}\right)=\frac{1}{\mathrm{c}}\left(\left(\frac{f^{\prime}}{r}\right)\left(-u_{x}\right)\right)+\frac{v_{x}}{\mathrm{c}}\left(\frac{f^{\prime}}{r}-\frac{f}{r^{2}}\right)+\frac{v_{x}}{\mathrm{c}^{2}}\left(\left(\frac{f^{\prime}}{r}\right)\left(-u_{x}\right)\right)
\end{aligned}
$$

If we put $v_{x}=u_{x}$, then

$$
\begin{gathered}
\frac{f^{\prime}}{r}-\frac{f}{r^{2}}=\frac{u_{x}}{\mathrm{c}}\left(\frac{f^{\prime}}{r}\right)-\frac{u_{x}}{\mathrm{c}}\left(\frac{f^{\prime}}{r}\right)+\frac{u_{x}}{\mathrm{c}}\left(\frac{f}{r^{2}}\right)+\frac{u_{x} u_{x}}{\mathrm{c}^{2}}\left(\frac{f^{\prime}}{r}\right) \\
-\left(\frac{f^{\prime}}{r}-\frac{f}{r^{2}}\right)=-\frac{u_{x}}{\mathrm{c}}\left(\frac{f}{r^{2}}\right)+\frac{u_{x}}{\mathrm{c}^{2}}\left(\left(\frac{f^{\prime}}{r}\right)\left(-u_{x}\right)\right) \\
\frac{f^{\prime}}{r}-\frac{f}{r^{2}}=\frac{u_{x}}{\mathrm{c}} \frac{f}{r^{2}}+\frac{u_{x} u_{x}}{\mathrm{c}^{2}} \frac{f^{\prime}}{r} \\
-\frac{f^{\prime}}{r}+\frac{f}{r^{2}}=-\frac{u_{x}}{\mathrm{c}} \frac{f}{r^{2}}-\frac{u_{x} u_{x}}{\mathrm{c}^{2}} \frac{f^{\prime}}{r}
\end{gathered}
$$

The equations are satisfied if

$$
u_{x}=-c
$$

Let us explain the minus sign for velocity. The choice of the observation point $x=r$ means that it lies to the right of the origin of coordinates. In the above calculations we used the definition of the vector product in the form 


$$
\begin{aligned}
& (\mathbf{u} \times \mathbf{B})_{x}=u_{y} B_{z}-u_{z} B_{y} \\
& (\mathbf{u} \times \mathbf{B})_{y}=u_{z} B_{x}-u_{x} B_{z} \\
& (\mathbf{u} \times \mathbf{B})_{z}=u_{x} B_{y}-u_{y} B_{x}
\end{aligned}
$$

and the definition of rotor

$$
\begin{aligned}
& (\operatorname{rot} \mathbf{B})_{x}=\frac{\partial B_{z}}{\partial y}-\frac{\partial B_{y}}{\partial z} \\
& (\operatorname{rot} \mathbf{B})_{y}=\frac{\partial B_{x}}{\partial z}-\frac{\partial B_{z}}{\partial x} \\
& (\operatorname{rot} \mathbf{B})_{z}=\frac{\partial B_{y}}{\partial x}-\frac{\partial B_{x}}{\partial y}
\end{aligned}
$$

Thus, our $(\mathbf{u} \times \mathbf{B})_{y}$ component is negative, and if the directions of the $x, y, z$ coordinate vectors form a right triplet, then the $y$-axis points away from us. The $x$-axis is directed to the right, the magnetic field is directed upward, and the electric field is directed toward us, in the negative $y$ direction. The vectors $\mathbf{E}, \mathbf{B}$ and $\mathbf{u}$ in the electromagnetic wave form the right triplet. Since $\mathbf{E}$ is directed toward us, $\mathbf{B}$ is directed upward, $\mathbf{u}$ is directed to the left, that is, the positive velocity direction is directed against the $x$-axis, respectively the negative velocity $u_{x}=-c$ means wave propagation to the right along the $x$-axis through point $x=r$, that is, outward of the sphere. Consequently, propagation of field excitation is possible only in one direction outward and only at the speed of light.

Note that a similar consideration for Maxwell's equations leads to

$$
\begin{gathered}
\left(\frac{f^{\prime}}{r}-\frac{f}{r^{2}}\right)=-\frac{1}{\mathrm{c}}\left(\left(\frac{f^{\prime}}{r}\right)\left(-u_{x}\right)\right) \\
\frac{f^{\prime}}{r}-\frac{f}{r^{2}}=\frac{u_{x}}{\mathrm{c}} \frac{f^{\prime}}{r}
\end{gathered}
$$

That is, there is no solution, Maxwell's equations do not describe the propagation of a spherical wave. The problems with the plane wave and spherical wave are commonly circumvented by transition from Maxwell's equations to wave equations, for which the plane wave propagates in both directions, but the spherical wave also propagates in both directions. The possibility of a spherical wave propagating inward is discarded without explanation. When passing from Maxwell's equations to the wave equations, the coincidence of the sets of their solutions is not proved.

After regrouping equations (8) and (9) we obtain

$$
\begin{aligned}
& \operatorname{rot}(\mathbf{E}-(\mathbf{v} \times \mathbf{B}))=-\frac{\partial}{\partial t}\left(\mathbf{B}+\frac{1}{c^{2}}(\mathbf{v} \times \mathbf{E})\right) \\
& c^{2} \operatorname{rot}\left(\mathbf{B}+\frac{1}{c^{2}}(\mathbf{v} \times \mathbf{E})\right)=\frac{\partial}{\partial t}(\mathbf{E}-(\mathbf{v} \times \mathbf{B}))+\frac{\hat{\mathbf{j}}}{\varepsilon_{0}}
\end{aligned}
$$

Let us apply the divergence operation to (10) and (11)

$$
\begin{gathered}
0=-\frac{\partial}{\partial t} \operatorname{div}\left(\mathbf{B}+\frac{1}{c^{2}}(\mathbf{v} \times \mathbf{E})\right) \\
0=\frac{\partial}{\partial t} \operatorname{div}(\mathbf{E}-(\mathbf{v} \times \mathbf{B}))+\operatorname{div} \frac{\hat{\mathbf{j}}}{\varepsilon_{0}}
\end{gathered}
$$

given the continuity equation for charge and current 


$$
\frac{\partial}{\partial t} \frac{\hat{\rho}}{\varepsilon_{0}}+\operatorname{div} \frac{\hat{\mathbf{j}}}{\varepsilon_{0}}=0
$$

we get

$$
\operatorname{div}(\mathbf{E}-(\mathbf{v} \times \mathbf{B}))=\frac{\hat{\rho}}{\varepsilon_{0}}
$$

that is to say $\frac{\partial}{\partial t}(\mathbf{E}-(\mathbf{v} \times \mathbf{B}))$ can traditionally be called the bias current, besides

$$
\operatorname{div}\left(\mathbf{B}+\frac{1}{c^{2}}(\mathbf{v} \times \mathbf{E})\right)=\text { const }
$$

Assuming that there are no magnetic charges, we get

$$
\operatorname{div}\left(\mathbf{B}+\frac{1}{c^{2}}(\mathbf{v} \times \mathbf{E})\right)=0
$$

Equations (10), (11), (12) and (13) will be called equations of electrodynamics. Taking into account (13), we can write

$$
\mathbf{B}+\frac{1}{c^{2}}(\mathbf{v} \times \mathbf{E})=\operatorname{rot} \widehat{\mathbf{A}}
$$

where $\widehat{\mathbf{A}}$ is the vector potential. Let us denote for convenience

$$
\begin{gathered}
\widehat{\mathbf{E}}=\mathbf{E}-(\mathbf{v} \times \mathbf{B}) \\
\widehat{\mathbf{B}}=\mathbf{B}+\frac{1}{c^{2}}(\mathbf{v} \times \mathbf{E})
\end{gathered}
$$

then

$$
\widehat{\mathbf{B}}=\operatorname{rot} \widehat{\mathbf{A}}
$$

and the equations of electrodynamics take the form

$$
\begin{aligned}
\operatorname{rot} \widehat{\mathbf{E}} & =-\frac{\partial \widehat{\mathbf{B}}}{\partial t} \\
c^{2} \operatorname{rot} \widehat{\mathbf{B}} & =\frac{\partial \widehat{\mathbf{E}}}{\partial t}+\frac{\hat{\mathbf{j}}}{\varepsilon_{0}} \\
\operatorname{div} \widehat{\mathbf{E}} & =\frac{\hat{\rho}}{\varepsilon_{0}} \\
\operatorname{div} \widehat{\mathbf{B}} & =0
\end{aligned}
$$

we have from (14)

$$
\operatorname{rot}\left(\widehat{\mathbf{E}}+\frac{\partial \widehat{\mathbf{A}}}{\partial t}\right)=\mathbf{0}
$$

so the expression in parentheses can be represented as a gradient of the scalar potential

$$
\begin{gathered}
\widehat{\mathbf{E}}+\frac{\partial \widehat{\mathbf{A}}}{\partial t}=-\operatorname{grad} \hat{\varphi} \\
\widehat{\mathbf{E}}=-\operatorname{grad} \hat{\varphi}-\frac{\partial \widehat{\mathbf{A}}}{\partial t}
\end{gathered}
$$

Next, using the standard scheme with the Lorentz calibration, we obtain the equations for the potentials

$$
\begin{aligned}
& \nabla^{2} \widehat{\mathbf{A}}-\frac{1}{c^{2}} \frac{\partial^{2} \widehat{\mathbf{A}}}{\partial t^{2}}=-\frac{\hat{\mathbf{j}}}{\varepsilon_{0} c^{2}} \\
& \nabla^{2} \hat{\varphi}-\frac{1}{c^{2}} \frac{\partial^{2} \hat{\varphi}}{\partial t^{2}}=-\frac{\hat{\rho}}{\varepsilon_{0}}
\end{aligned}
$$




$$
\operatorname{div} \widehat{\mathbf{A}}=-\frac{1}{c^{2}} \frac{\partial \hat{\varphi}}{\partial t}
$$

The solutions of these equations are known; knowing the potentials, we will find the solutions for

$$
\begin{gathered}
\widehat{\mathbf{E}}=\mathbf{E}-(\mathbf{v} \times \mathbf{B}) \\
\widehat{\mathbf{B}}=\mathbf{B}+\frac{1}{c^{2}}(\mathbf{v} \times \mathbf{E})
\end{gathered}
$$

Now we need to find $\mathrm{E}$ and $\mathrm{B}$ from these equations. For the special case where the velocity is directed along the $x$-axis and the fields are orthogonal to it and to each other, we get

$$
\begin{gathered}
\hat{E}_{y}=E_{y}-\left(-v_{x} B_{z}\right) \\
\hat{B}_{z}=B_{z}+\frac{1}{c^{2}} v_{x} E_{y} \\
\hat{E}_{y}=E_{y}+v_{x} B_{z} \\
\hat{B}_{z}=B_{z}+\frac{1}{c^{2}} v_{x} E_{y} \\
B_{z}=\hat{B}_{z}-\frac{1}{c^{2}} v_{x} E_{y} \\
\hat{E}_{y}=E_{y}+v_{x} \hat{B}_{z}-\frac{1}{c^{2}} v_{x}^{2} E_{y} \\
E_{y}-\frac{1}{c^{2}} v_{x}{ }^{2} E_{y}=\hat{E}_{y}-v_{x} \hat{B}_{z} \\
E_{y}=\frac{\hat{E}_{y}-v_{x} \hat{B}_{z}}{1-v_{x}^{2} / c^{2}}
\end{gathered}
$$

For our special case of transverse fields, we have

similarly

$$
\mathbf{E}=\frac{\widehat{\mathbf{E}}+(\mathbf{v} \times \widehat{\mathbf{B}})}{1-v^{2} / c^{2}}
$$

$$
\begin{gathered}
\hat{E}_{y}=E_{y}+v_{x} B_{z} \\
\hat{B}_{z}=B_{z}+\frac{1}{c^{2}} v_{x} E_{y} \\
E_{y}=\hat{E}_{y}-v_{x} B_{z} \\
\hat{B}_{z}=B_{z}+\frac{1}{c^{2}} v_{x}\left(\hat{E}_{y}-v_{x} B_{z}\right) \\
\hat{B}_{z}=B_{z}+\frac{1}{c^{2}} v_{x} \hat{E}_{y}-\frac{1}{c^{2}} v_{x}^{2} B_{z} \\
B_{z}\left(1-\frac{1}{c^{2}} v_{x}^{2}\right)=\hat{B}_{z}-\frac{1}{c^{2}} v_{x} \hat{E}_{y} \\
B_{z}=\frac{\hat{B}_{z}-\frac{1}{c^{2}} v_{x} \hat{E}_{y}}{1-v_{x}^{2} / c^{2}} \\
\mathbf{B}=\frac{\widehat{\mathbf{B}}-\frac{1}{c^{2}}(\mathbf{v} \times \mathbf{\mathbf { E }})}{1-v^{2} / c^{2}}
\end{gathered}
$$

Consider the following example. Let a stationary charge $q$ be located at the origin of coordinates in some reference frame, and choose a point with coordinates $x=0, y=0, z \neq 0$ moving parallel to the $x$-axis in its negative direction with speed $\mathbf{v}$ as an observation point. The 
magnetic field of the charge is zero, the electric field equal to the gradient of the scalar potential is known from electrostatics

$$
\hat{E}_{z}=\frac{q}{4 \pi \varepsilon_{0} z^{2}}
$$

In our case

$$
\begin{aligned}
& \hat{\mathbf{E}}=\mathbf{E}-(\mathbf{v} \times \mathbf{B}) \\
& \mathbf{0}=\mathbf{B}+\frac{1}{c^{2}}(\mathbf{v} \times \mathbf{E}) \\
& \hat{\mathbf{E}}=\mathbf{E}+\left(\mathbf{v} \times \frac{1}{c^{2}}(\mathbf{v} \times \mathbf{E})\right)=\mathbf{E}+\frac{1}{c^{2}}(\mathbf{v} \times(\mathbf{v} \times \mathbf{E}))=\mathbf{E}+\frac{1}{c^{2}}(\mathbf{v}(\mathbf{v} \cdot \mathbf{E})-\mathbf{E}(\mathbf{v} \cdot \mathbf{v}))=\mathbf{E}-\frac{v^{2}}{c^{2}} \mathbf{E} \\
&=\mathbf{E}\left(1-\frac{v^{2}}{c^{2}}\right) \\
& \mathbf{E}=\frac{\hat{\mathbf{E}}}{1-v^{2} / c^{2}} \\
& E_{z}=\frac{q}{4 \pi \varepsilon_{0} z^{2}} \frac{1}{1-v^{2} / c^{2}}
\end{aligned}
$$

Now let the charge at the origin move along the $x$-axis in its positive direction with velocity $\mathbf{v}$ in the same frame of reference, and let the observation point with the former coordinates be stationary. According to, for example, [2] (Chapter 26, paragraph 2, p. 267) the field calculated from the potential is

$$
\hat{E}_{z}=\frac{q}{4 \pi \varepsilon_{0} z^{2}} \frac{1}{\sqrt{1-v^{2} / c^{2}}}
$$

The observation point is stationary, so $E_{z}=\hat{E}_{z}$.

In the two considered examples we have two different results, which should be the same. It follows that the correct transformations are as follows

and the inverse has the form

$$
\begin{gathered}
\widehat{\mathbf{E}}=\frac{\mathbf{E}-(\mathbf{v} \times \mathbf{B})}{\sqrt{1-v^{2} / c^{2}}} \\
\widehat{\mathbf{B}}=\frac{\mathbf{B}+\frac{1}{c^{2}}(\mathbf{v} \times \mathbf{E})}{\sqrt{1-v^{2} / c^{2}}}
\end{gathered}
$$

$$
\begin{array}{r}
\mathbf{E}=\frac{\widehat{\mathbf{E}}+(\mathbf{v} \times \widehat{\mathbf{B}})}{\sqrt{1-v^{2} / c^{2}}} \\
\mathbf{B}=\frac{\widehat{\mathbf{B}}-\frac{1}{c^{2}}(\mathbf{v} \times \widehat{\mathbf{E}})}{\sqrt{1-v^{2} / c^{2}}}
\end{array}
$$

The equations of electrodynamics thus take the form

$$
\begin{aligned}
& \operatorname{rot}\left(\frac{\mathbf{E}-(\mathbf{v} \times \mathbf{B})}{\sqrt{1-v^{2} / c^{2}}}\right)=-\frac{\partial}{\partial t}\left(\frac{\mathbf{B}+\frac{1}{c^{2}}(\mathbf{v} \times \mathbf{E})}{\sqrt{1-v^{2} / c^{2}}}\right) \\
& c^{2} \operatorname{rot}\left(\frac{\mathbf{B}+\frac{1}{c^{2}}(\mathbf{v} \times \mathbf{E})}{\sqrt{1-v^{2} / c^{2}}}\right)=\frac{\partial}{\partial t}\left(\frac{\mathbf{E}-(\mathbf{v} \times \mathbf{B})}{\sqrt{1-v^{2} / c^{2}}}\right)+\frac{\hat{\mathbf{j}}}{\varepsilon_{0}}
\end{aligned}
$$




$$
\begin{gathered}
\operatorname{div}\left(\frac{\mathbf{E}-(\mathbf{v} \times \mathbf{B})}{\sqrt{1-v^{2} / c^{2}}}\right)=\frac{\hat{\rho}}{\varepsilon_{0}} \\
\operatorname{div}\left(\frac{\mathbf{B}+\frac{1}{c^{2}}(\mathbf{v} \times \mathbf{E})}{\sqrt{1-v^{2} / c^{2}}}\right)=0
\end{gathered}
$$

the bias current is $\frac{\partial}{\partial t}\left(\frac{\mathbf{E}-(\mathbf{v} \times \mathbf{B})}{\sqrt{1-v^{2} / c^{2}}}\right)$.

All the reasoning from the beginning of the article, with the inclusion of the multiplier $\frac{1}{\sqrt{1-v^{2} / c^{2}}}$ remains valid; in particular, the plane and spherical wave are solutions of the equations in the absence of currents and charges, since the multiplier $\frac{1}{\sqrt{1-v^{2} / c^{2}}}$ is taken out of all derivatives due to the constancy of $\mathbf{v} . \mathbf{v}$ is simply a parameter of the equation, which, although it may take different values for different fields, is considered constant and the derivatives are not taken from it. Wave equations with sources for vector and scalar potentials are still true, and fields are expressed through them by formulas

$$
\begin{gathered}
\frac{\mathbf{B}+\frac{1}{c^{2}}(\mathbf{v} \times \mathbf{E})}{\sqrt{1-v^{2} / c^{2}}}=\operatorname{rot} \widehat{\mathbf{A}} \\
\frac{\mathbf{E}-(\mathbf{v} \times \mathbf{B})}{\sqrt{1-v^{2} / c^{2}}}=-\operatorname{grad} \hat{\varphi}-\frac{\partial \widehat{\mathbf{A}}}{\partial t}
\end{gathered}
$$

Let us return to the above example, where charge $q$ is located at the origin, but let it now move along the $x$-axis in its positive direction with velocity $\mathbf{v}_{1}$. As an observation point we again choose a point with coordinates $x=0, y=0, z \neq 0$, moving parallel to the $x$-axis in its negative direction with velocity $\mathbf{v}_{2}$. Again we have

$$
\hat{E}_{z}=\frac{q}{4 \pi \varepsilon_{0} z^{2}} \frac{1}{\sqrt{1-v_{1}^{2} / c^{2}}}
$$

Using (20) and (21), we can find both the magnetic field and the $E_{z}$ field, the formula for which includes velocity $\mathbf{v}_{2}$ and velocity $\mathbf{v}_{1}$.

$$
\begin{gathered}
\sqrt{1-v_{2}^{2} / c^{2}} \mathbf{E}=\hat{\mathbf{E}}+\left(\mathbf{v}_{2} \times \widehat{\mathbf{B}}\right)=\hat{\mathbf{E}}+\frac{1}{c^{2}}\left(\mathbf{v}_{2} \times\left(\mathbf{v}_{1} \times \widehat{\mathbf{E}}\right)\right)=\hat{\mathbf{E}}+\frac{1}{c^{2}}\left(\mathbf{v}_{1}\left(\mathbf{v}_{2} \cdot \widehat{\mathbf{E}}\right)-\widehat{\mathbf{E}}\left(\mathbf{v}_{1} \cdot \mathbf{v}_{2}\right)\right) \\
=\hat{\mathbf{E}}-\frac{\left(\mathbf{v}_{1} \cdot \mathbf{v}_{2}\right)}{c^{2}} \hat{\mathbf{E}}=\hat{\mathbf{E}}\left(1-\frac{\left(\mathbf{v}_{1} \cdot \mathbf{v}_{2}\right)}{c^{2}}\right) \\
\sqrt{1-v_{2}^{2} / c^{2}} E_{z}=\frac{q}{4 \pi \varepsilon_{0} z^{2}} \frac{1}{\sqrt{1-v_{1}^{2} / c^{2}}}\left(1+\frac{v_{1} v_{2}}{c^{2}}\right)
\end{gathered}
$$

Consider the same configuration, but let the charge rest at the origin and the observation point has a velocity parallel to the $x$-axis in its negative direction $\mathbf{v}_{3}$. We also know the field $E_{z}$ for it, it depends only on $\mathbf{v}_{3}$.

$$
E_{z}=\frac{q}{4 \pi \varepsilon_{0} z^{2}} \frac{1}{\sqrt{1-v_{3}^{2} / c^{2}}}
$$

By equating the $E_{z}$ fields in these two configurations, we express the velocity $\mathbf{v}_{3}$ through the velocities $\mathbf{v}_{1}$ and $\mathbf{v}_{2}$.

$$
\frac{1}{\sqrt{1-v_{3}^{2} / c^{2}}}=\frac{\left(1+\frac{v_{1} v_{2}}{c^{2}}\right)}{\sqrt{1-v_{2}^{2} / c^{2}} \sqrt{1-v_{1}^{2} / c^{2}}}
$$




$$
v_{3}^{2} / c^{2}=1-\frac{\left(1-v_{2}^{2} / c^{2}\right)\left(1-v_{1}^{2} / c^{2}\right)}{\left(1+\frac{v_{1} v_{2}}{c^{2}}\right)^{2}}
$$

We can call this expression the velocity transformation formula for electrodynamics. If $\mathbf{v}_{1}$ and $\mathbf{v}_{2}$ are close in modulo to the speed of light, $\mathbf{v}_{3}$ is less than the speed of light. The result is not related to Lorentz transformations; it follows from the equations of electrodynamics. On the basis of them we cannot say that the relative velocity of the charge and the observation point cannot exceed the speed of light, but we can say that in any case the fields at the observation point are obtained the same as when the observation point moves relative to a stationary charge at some speed not exceeding the speed of light. We may verify that the velocity is the same as that obtained by the addition of velocities to the Lorentz transformation for this case

$$
v_{3}=\frac{v_{1}+v_{2}}{1+\frac{v_{1} v_{2}}{c^{2}}}
$$

Note that we consider the fields in a single three-dimensional coordinate system, without transformations to another coordinate system.

Let us write down the equations in a more convenient system of units, in which $c=1$.

$$
\begin{aligned}
\operatorname{rot}(\gamma(\mathbf{E}-(\mathbf{v} \times \mathbf{B}))) & =-\frac{\partial}{\partial t}(\gamma(\mathbf{B}+(\mathbf{v} \times \mathbf{E}))) \\
\operatorname{rot}(\gamma(\mathbf{B}+(\mathbf{v} \times \mathbf{E}))) & =\frac{\partial}{\partial t}(\gamma(\mathbf{E}-(\mathbf{v} \times \mathbf{B})))+4 \pi \hat{\mathbf{j}} \\
\gamma & =\frac{1}{\sqrt{1-v^{2}}}
\end{aligned}
$$

Let us check, whether these equations satisfy the fields of the charge moving uniformly with speed $\mathbf{u}$. Let us say at once that they do not satisfy in this form, but they can be modified and written in the form

$$
\begin{aligned}
\operatorname{rot}(\gamma(\mathbf{E}-(\mathbf{v} \times \mathbf{B}))-\Gamma \mathbf{v}(\mathbf{v} \cdot \mathbf{E})) & =-\frac{\partial}{\partial t}(\gamma(\mathbf{B}+(\mathbf{v} \times \mathbf{E}))-\Gamma \mathbf{v}(\mathbf{v} \cdot \mathbf{B})) \\
\operatorname{rot}(\gamma(\mathbf{B}+(\mathbf{v} \times \mathbf{E}))-\Gamma \mathbf{v}(\mathbf{v} \cdot \mathbf{B})) & =\frac{\partial}{\partial t}(\gamma(\mathbf{E}-(\mathbf{v} \times \mathbf{B}))-\Gamma \mathbf{v}(\mathbf{v} \cdot \mathbf{E}))+4 \pi \hat{\mathbf{j}} \\
\Gamma & =\frac{\gamma-1}{v^{2}}
\end{aligned}
$$

Denote by $\mathbf{E}_{s}$ the electric field of the stationary charge, its magnetic field is zero $\mathbf{B}_{s}=\mathbf{0}$. The stationary charge is at the same point at which the moving charge is at a given time. Let us express the fields of the moving charge with velocity $\mathbf{u}$ in the form

$$
\begin{aligned}
& \mathbf{E}=\gamma\left(\mathbf{E}_{s}-\left(\mathbf{u} \times \mathbf{B}_{s}\right)\right)-\Gamma \mathbf{u}\left(\mathbf{u} \cdot \mathbf{E}_{s}\right)=\gamma \mathbf{E}_{s}-\Gamma \mathbf{u}\left(\mathbf{u} \cdot \mathbf{E}_{s}\right) \\
& \mathbf{B}=\gamma\left(\mathbf{B}_{s}+\left(\mathbf{u} \times \mathbf{E}_{s}\right)\right)-\Gamma \mathbf{u}\left(\mathbf{u} \cdot \mathbf{B}_{s}\right)=\gamma\left(\mathbf{u} \times \mathbf{E}_{s}\right)
\end{aligned}
$$

Let's substitute these fields into the expressions included in equations (26) and (27) by putting $\mathbf{v}=-\mathbf{u}$ 


$$
\begin{aligned}
\gamma(\mathbf{E}-(\mathbf{v} \times \mathbf{B})) & -\Gamma \mathbf{v}(\mathbf{v} \cdot \mathbf{E})=\gamma(\mathbf{E}+(\mathbf{u} \times \mathbf{B}))-\Gamma \mathbf{u}(\mathbf{u} \cdot \mathbf{E}) \\
& =\gamma\left(\gamma \mathbf{E}_{s}-\Gamma \mathbf{u}\left(\mathbf{u} \cdot \mathbf{E}_{s}\right)\right)+\gamma\left(\mathbf{u} \times \gamma\left(\mathbf{u} \times \mathbf{E}_{s}\right)\right)-\Gamma \mathbf{u}\left(\mathbf{u} \cdot\left(\gamma \mathbf{E}_{s}-\Gamma \mathbf{u}\left(\mathbf{u} \cdot \mathbf{E}_{s}\right)\right)\right) \\
& =\gamma^{2} \mathbf{E}_{s}-\gamma \Gamma \mathbf{u}\left(\mathbf{u} \cdot \mathbf{E}_{s}\right)+\gamma^{2}\left(\mathbf{u} \times\left(\mathbf{u} \times \mathbf{E}_{s}\right)\right)-\Gamma \mathbf{u}\left(\mathbf{u} \cdot\left(\gamma \mathbf{E}_{s}-\Gamma \mathbf{u}\left(\mathbf{u} \cdot \mathbf{E}_{s}\right)\right)\right) \\
& =\gamma^{2} \mathbf{E}_{s}-\gamma \Gamma \mathbf{u}\left(\mathbf{u} \cdot \mathbf{E}_{s}\right)+\gamma^{2}\left(\mathbf{u} \times\left(\mathbf{u} \times \mathbf{E}_{s}\right)\right)-\gamma \Gamma \mathbf{u}\left(\mathbf{u} \cdot \mathbf{E}_{s}\right) \\
& +\Gamma \Gamma \mathbf{u}\left(\mathbf{u} \cdot\left(\mathbf{u}\left(\mathbf{u} \cdot \mathbf{E}_{s}\right)\right)\right) \\
& =\gamma^{2} \mathbf{E}_{s}-\gamma \Gamma \mathbf{u}\left(\mathbf{u} \cdot \mathbf{E}_{s}\right)+\gamma^{2} \mathbf{u}\left(\mathbf{u} \times \mathbf{E}_{s}\right)-\gamma^{2} \mathbf{E}_{s}(\mathbf{u} \cdot \mathbf{u})-\gamma \Gamma \mathbf{u}\left(\mathbf{u} \cdot \mathbf{E}_{s}\right) \\
& +\Gamma \Gamma \mathbf{u}\left(\mathbf{u} \cdot \mathbf{E}_{s}\right)(\mathbf{u} \cdot \mathbf{u}) \\
& =\gamma^{2} \mathbf{E}_{s}-\gamma \Gamma \mathbf{u}\left(\mathbf{u} \cdot \mathbf{E}_{s}\right)+\gamma^{2} \mathbf{u}\left(\mathbf{u} \times \mathbf{E}_{s}\right)-\gamma^{2} \mathbf{E}_{s}(\mathbf{u} \cdot \mathbf{u}) \\
& -\Gamma \mathbf{u}\left(\mathbf{u} \cdot \mathbf{E}_{s}\right)(\gamma-\Gamma(\mathbf{u} \cdot \mathbf{u})) \\
& =\gamma^{2}(1-(\mathbf{u} \cdot \mathbf{u})) \mathbf{E}_{s}-\gamma^{2} \mathbf{E}_{s}-\gamma \Gamma \mathbf{u}\left(\mathbf{u} \cdot \mathbf{E}_{s}\right)+\gamma^{2} \mathbf{u}\left(\mathbf{u} \times \mathbf{E}_{s}\right)-\Gamma \mathbf{u}\left(\mathbf{u} \cdot \mathbf{E}_{s}\right) \\
& =\mathbf{E}_{s}-\gamma \Gamma \mathbf{u}\left(\mathbf{u} \cdot \mathbf{E}_{s}\right)+\gamma^{2} \mathbf{u}\left(\mathbf{u} \cdot \mathbf{E}_{s}\right)-\Gamma \mathbf{u}\left(\mathbf{u} \cdot \mathbf{E}_{s}\right)=\mathbf{E}_{s}+\mathbf{u}\left(\mathbf{u} \cdot \mathbf{E}_{s}\right)\left(\gamma^{2}-\gamma \Gamma-\Gamma\right) \\
& =\mathbf{E}_{s}
\end{aligned}
$$

Here it is taken into account that

$$
\begin{gathered}
\gamma \gamma(1-(\mathbf{u} \cdot \mathbf{u}))=1 \quad \gamma-\Gamma(\mathbf{u} \cdot \mathbf{u})=1 \\
\gamma^{2}-\gamma \Gamma-\Gamma=\gamma^{2}-\frac{\gamma(\gamma-1)}{u^{2}}-\frac{(\gamma-1)}{u^{2}}=\frac{\gamma^{2} u^{2}-\gamma^{2}+\gamma-\gamma+1}{u^{2}}=\frac{\gamma^{2} u^{2}-\gamma^{2}+1}{u^{2}} \\
=\frac{\frac{1}{1-u^{2}} u^{2}-\frac{1}{1-u^{2}}+1}{u^{2}}=\frac{1}{1-u^{2}}-\frac{1}{\left(1-u^{2}\right) u^{2}}+\frac{1}{u^{2}}=\frac{u^{2}-1+1-u^{2}}{\left(1-u^{2}\right) u^{2}}=0 \\
\gamma(\mathbf{B}+(\mathbf{v} \times \mathbf{E}))-\Gamma \mathbf{v}(\mathbf{v} \cdot \mathbf{B})=\gamma(\mathbf{B}-(\mathbf{u} \times \mathbf{E}))-\Gamma \mathbf{u}(\mathbf{u} \cdot \mathbf{B}) \\
=\gamma\left(\gamma\left(\mathbf{u} \times \mathbf{E}_{s}\right)-\left(\mathbf{u} \times\left(\gamma \mathbf{E}_{s}-\Gamma \mathbf{u}\left(\mathbf{u} \cdot \mathbf{E}_{s}\right)\right)\right)-\Gamma \mathbf{u}\left(\mathbf{u} \cdot\left(\gamma\left(\mathbf{u} \times \mathbf{E}_{s}\right)\right)\right)\right. \\
=\gamma\left(\gamma\left(\mathbf{u} \times \mathbf{E}_{s}\right)-\gamma\left(\mathbf{u} \times \mathbf{E}_{s}\right)\right)-\gamma \Gamma \mathbf{u}\left(\mathbf{u} \cdot\left(\mathbf{u} \times \mathbf{E}_{s}\right)\right)=\mathbf{0}
\end{gathered}
$$

Since the rotor of the electric field of a stationary charge and its time derivative are zero, the field of a uniformly moving charge is indeed a solution of equations (26) and (27) at zero current, that is, at points where there is no charge.

For points where there is a non-zero charge density a separate consideration is necessary, we will return to it a little later.

Thus, it is necessary to write the previously introduced notations in the augmented form

$$
\begin{aligned}
& \widehat{\mathbf{E}}=\gamma(\mathbf{E}-(\mathbf{v} \times \mathbf{B}))-\Gamma \mathbf{v}(\mathbf{v} \cdot \mathbf{E}) \\
& \widehat{\mathbf{B}}=\gamma(\mathbf{B}+(\mathbf{v} \times \mathbf{E}))-\Gamma \mathbf{v}(\mathbf{v} \cdot \mathbf{B})
\end{aligned}
$$

One can check that these transformations form a group with the corresponding velocity addition formula.

If at a particular point in space there is an electric $\mathbf{E}$ and a magnetic $\mathbf{B}$ field at a particular fixed point in space and at the same point in space there is an observation point moving with constant velocity $\mathbf{v}$, then the magnitudes of the electric $\mathbf{E}_{\mathbf{v}}$ and magnetic $\mathbf{B}_{\mathbf{v}}$ for it are described by the following expressions

$$
\begin{aligned}
& \mathbf{E}_{\mathbf{v}}=\gamma(\mathbf{E}+(\mathbf{v} \times \mathbf{B}))-\Gamma \mathbf{v}(\mathbf{v} \cdot \mathbf{E}) \\
& \mathbf{B}_{\mathbf{v}}=\gamma(\mathbf{B}-(\mathbf{v} \times \mathbf{E}))-\Gamma \mathbf{v}(\mathbf{v} \cdot \mathbf{B})
\end{aligned}
$$

If there is a charge in the moving observation point, only the electric field $\mathbf{E}_{\mathbf{v}}$ acts on it. In general, it is reasonable to assume that only the electric field acts on charges and never the magnetic field. If the point in which the charge is located moves, then the magnetic field simply 
gives an additional contribution to the electric field. Then there is no need to use the concept of the Lorentz force.

In particular, if the fields are created by a charge moving with velocity $\mathbf{u}$, then

and, as shown above, at $\mathbf{v}=\mathbf{u}$

$$
\begin{aligned}
& \mathbf{E}=\gamma \mathbf{E}_{s}-\Gamma \mathbf{u}\left(\mathbf{u} \cdot \mathbf{E}_{s}\right) \\
& \mathbf{B}=\gamma\left(\mathbf{u} \times \mathbf{E}_{s}\right)
\end{aligned}
$$

$$
\begin{aligned}
& \mathbf{E}_{\mathbf{v}}=\mathbf{E}_{S} \\
& \mathbf{B}_{\mathbf{v}}=\mathbf{0}
\end{aligned}
$$

It turns out that if the velocity of the observation point is equal to the velocity of the charge, then the field in it coincides with the field of a stationary charge, which is natural, because the charge and the observation point can be stationary relative to each other and observed in a uniformly moving frame of reference.

If at a certain point of time there is an electric $\mathbf{E}$ and a magnetic $\mathbf{B}$ field at a particular uniformly moving observation point, and at the same point of space there is also another observation point moving with a constant, but different speed, then by means of a pair of consecutive transformations we can first convert the fields at the first observation point into fields for the stationary observation point, and then from them we obtain fields for the second moving observation point. In view of group properties, we can replace these two transformations by one equal to their product.

Returning to equations (26) and (27) for the case when there is a non-zero charge density, we see that if the charge creating the field moves uniformly, and the observation point moves uniformly at the same speed, and the coordinates of the charge coincide with the observation point, then for this observation point the charge is stationary and the current density at this point is zero. Thus, the field of a moving charge is a solution of equations (26) and (27) and in the case of a nonzero charge density.

If the charge and the observation point are still stationary relative to each other, but are considered in a frame of reference that moves unevenly, then the charge fields will no longer satisfy equations (26) and (27) for any parameter $v$. With uniform motion, $v$ is equal to the velocity of the charge at some previous moment in time, at which the acceleration of the charge was zero. But if the acceleration at that moment was nonzero, then the equations should include terms with the acceleration dimension. It can be assumed that in this case, instead of converting (28) and (29), more general transformations should be substituted into the equations, which also depend on the parameter that makes sense of acceleration. It can also be assumed that these transformations should also form a group, but already two-parameter.

Consider a system of two identical charges. Their interaction does not depend on the frame of reference from which we observe them. If, for example, they are stationary relative to each other, their interaction describes Coulomb's law. If we observe them from an inertial frame of reference, each of them moves uniformly and creates corresponding fields. If we observe them from a non-inertial system, each of them will emit electromagnetic waves. But with any complex nature of the motion of the reference frame, their interaction is ultimately described by Coulomb's law for electrostatics.

Let there be a fixed charge and a fixed observation point. We will consider them in an arbitrary coordinate system, in which both the charge and the observation point will move in the same way and in general accelerated. In this point moving synchronously with the charge, we observe an electrostatic Coulomb field. At the observation point with the same coordinates, 
which is stationary, the fields of a moving charge are observed. We would like to find field transformations between stationary and moving observation points that include free parameters with the dimension of velocity and acceleration. Substituting these transformations into the equations of electrodynamics, we would make them universal. The movement of each individual charge can be compensated by the same movement of the observation point, but we want the field transformations to automatically compensate for the movement of any charge with the appropriate choice of values of free parameters. We have a one-parameter group of field transformations with the velocity parameter $\mathrm{v}$, and we would like to find a two-parameter group of field transformations in which the second parameter would make sense of acceleration. Then we will substitute the transformation that leads the fields corresponding to arbitrary velocity and acceleration to fields with zero values of those into the equations. We would like to find such a group together with mathematicians, or prove that it does not exist, which would be strange, because fields of arbitrarily moving charges exist in nature and obey certain equations. At the same time, it is enough to find a group specifically for converting fields, it is not necessary that any coordinate transformations correspond to it.

It may be possible to find such a group using a direct search with symbolic calculations. Once the two-parameter group of transformations is found, we can say that all frames of reference are equal, not only inertial ones. The principle of relativity will become absolute.

Consider the well-known formulas for the fields of an arbitrarily moving charge, presented, in particular, in [3] (Chapter 5). The electric and magnetic field of a stationary charge $Q$ has the form

$$
\mathbf{E}_{\mathrm{s}}=Q \frac{\mathbf{r}}{r^{3}} \quad \mathbf{B}_{\mathrm{s}}=\mathbf{0}
$$

where $\mathbf{r}$ is the vector from the location of the charge to the observation point.

The field of a uniformly moving charge located at the same point as a stationary one

$$
\begin{aligned}
\mathbf{E}=\gamma \mathbf{E}_{s}-\Gamma \mathbf{u}\left(\mathbf{u} \cdot \mathbf{E}_{s}\right) & =\gamma Q \frac{\mathbf{r}}{r^{3}}-\Gamma \mathbf{u}\left(\mathbf{u} \cdot Q \frac{\mathbf{r}}{r^{3}}\right) \\
\mathbf{B}=\gamma\left(\mathbf{u} \times \mathbf{E}_{s}\right) & =(\mathbf{u} \times \mathbf{E})
\end{aligned}
$$

Another representation of these fields is given in [3] (Chapter 5).

$$
\begin{aligned}
\mathbf{E}=\gamma \mathbf{E}_{s}-\Gamma \mathbf{u}\left(\mathbf{u} \cdot \mathbf{E}_{s}\right) & =\frac{\gamma Q \hat{\mathbf{r}}}{\left(\hat{r}^{2}+\gamma^{2}(\mathbf{u} \cdot \hat{\mathbf{r}})^{2}\right)^{3 / 2}} \\
\mathbf{B}= & (\mathbf{u} \times \mathbf{E})
\end{aligned}
$$

where

$$
\begin{gathered}
\hat{\mathbf{r}}=\gamma \mathbf{r}-\Gamma \mathbf{u}(\mathbf{u} \cdot \mathbf{r}) \\
r^{2}=\hat{r}^{2}+\gamma^{2}(\mathbf{u} \cdot \hat{\mathbf{r}})^{2}
\end{gathered}
$$

Using $\hat{\mathbf{r}}$ in [3] (Chapter 5), the fields of an arbitrarily moving charge are represented as

$$
\begin{gathered}
\mathbf{E}=Q \frac{\gamma \hat{\mathbf{r}}}{\left(\hat{r}^{2}+\gamma^{2}(\mathbf{U} \cdot \hat{\mathbf{r}})^{2}\right)^{3 / 2}}+Q \frac{\gamma^{3}(\widehat{\mathbf{R}} \times(\hat{\mathbf{r}} \times \mathbf{W}))}{\left(\hat{r}^{2}+\gamma^{2}(\mathbf{U} \cdot \hat{\mathbf{r}})^{2}\right)^{3 / 2}} \\
\mathbf{B}=(\mathbf{N} \times \mathbf{E})
\end{gathered}
$$

where we are looking for the fields at the stationary observation point at the current time $t$, $\mathbf{U}$ and $\mathbf{W}$ denote the velocity and acceleration of the charge at some earlier time moment $T$, characterized by the fact that at that moment the charge was at a point from which light with unit speed in our consideration reached in time $\widehat{R}=t-T$ the observation point, vector $\widehat{\mathbf{R}}$ drawn from that point in the past to the observation point. The vector $\widehat{\mathbf{R}}$ is defined by the formula 


$$
\begin{aligned}
\widehat{\mathbf{R}} & =\hat{\mathbf{r}}+\mathbf{U} \hat{R} \\
\mathbf{N} & =\frac{\widehat{\mathbf{R}}}{\hat{R}} \\
\hat{\mathbf{r}} & =\gamma \mathbf{r}-\Gamma \mathbf{U}(\mathbf{U} \cdot \mathbf{r}) \\
r^{2} & =\hat{r}^{2}+\gamma^{2}(\mathbf{U} \cdot \widehat{\mathbf{r}})^{2} \\
\gamma & =\frac{1}{\sqrt{1-U^{2}}}
\end{aligned}
$$

Let's express the fields through $\mathbf{E}_{\mathbf{U}}$, denoting the electric field of a charge moving uniformly with speed $\mathbf{U}$ and having the form

$$
\mathbf{E}_{\mathbf{U}}=\gamma \mathbf{E}_{\mathrm{s}}-\Gamma \mathbf{U}\left(\mathbf{U} \cdot \mathbf{E}_{\mathrm{s}}\right)=Q \frac{\gamma \hat{\mathbf{r}}}{\left(\hat{r}^{2}+\gamma^{2}(\mathbf{U} \cdot \hat{\mathbf{r}})^{2}\right)^{3 / 2}}
$$

It is assumed that both the stationary charge and the uniformly moving charge are at the point from which vector $\hat{\mathbf{r}}$ is drawn to the observation point. This point is defined by the vector $\hat{R}$, that is, it is the point where the charge would be at moment $t$ if it were moving uniformly with speed $\mathbf{U}$ after moment $T$.

$$
\begin{aligned}
& \mathbf{E}=Q \frac{\gamma \hat{\mathbf{r}}}{\left(\hat{r}^{2}+\gamma^{2}(\mathbf{U} \cdot \hat{\mathbf{r}})^{2}\right)^{3 / 2}}+Q \frac{\gamma^{3}(\widehat{\mathbf{R}} \times(\hat{\mathbf{r}} \times \mathbf{W}))}{\left(\hat{r}^{2}+\gamma^{2}(\mathbf{U} \cdot \hat{\mathbf{r}})^{2}\right)^{3 / 2}} \\
& =Q \frac{\gamma \hat{\mathbf{r}}}{\left(\hat{r}^{2}+\gamma^{2}(\mathbf{U} \cdot \hat{\mathbf{r}})^{2}\right)^{3 / 2}}+Q \frac{\gamma^{2}(\widehat{\mathbf{R}} \times(\gamma \hat{\mathbf{r}} \times \mathbf{W}))}{\left(\hat{r}^{2}+\gamma^{2}(\mathbf{U} \cdot \hat{\mathbf{r}})^{2}\right)^{3 / 2}}=\mathbf{E}_{\mathbf{U}}+\gamma^{2}\left(\widehat{\mathbf{R}} \times\left(\mathbf{E}_{\mathbf{U}} \times \mathbf{W}\right)\right) \\
& =\mathbf{E}_{\mathbf{U}}\left(1+\gamma^{2}(\widehat{\mathbf{R}} \cdot \mathbf{W})\right)-\gamma^{2} \mathbf{W}\left(\widehat{\mathbf{R}} \cdot \mathbf{E}_{\mathbf{U}}\right) \\
& \mathbf{B}=\frac{1}{\hat{R}}(\widehat{\mathbf{R}} \times \mathbf{E})=\frac{1}{\hat{R}}\left(\widehat{\mathbf{R}} \times \mathbf{E}_{\mathbf{U}}\right)+\gamma^{2} \frac{1}{\hat{R}}\left(\widehat{\mathbf{R}} \times\left(\widehat{\mathbf{R}} \times\left(\mathbf{E}_{\mathbf{U}} \times \mathbf{W}\right)\right)\right) \\
& \frac{1}{\hat{R}}\left(\widehat{\mathbf{R}} \times \mathbf{E}_{\mathbf{U}}\right)=\frac{1}{\hat{R}}\left((\hat{\mathbf{r}}+\mathbf{U} \hat{R}) \times\left(Q \frac{\gamma \hat{\mathbf{r}}}{\left(\hat{r}^{2}+\gamma^{2}(\mathbf{U} \cdot \hat{\mathbf{r}})^{2}\right)^{3 / 2}}\right)\right)=\frac{1}{\hat{R}}\left(\mathbf{U} \hat{R} \times \mathbf{E}_{\mathbf{U}}\right)=\left(\mathbf{U} \times \mathbf{E}_{\mathbf{U}}\right)=\mathbf{B}_{\mathbf{U}} \\
& \mathbf{B}=\frac{1}{\widehat{R}}(\widehat{\mathbf{R}} \times \mathbf{E})=\mathbf{B}_{\mathbf{U}}\left(1+\gamma^{2}(\widehat{\mathbf{R}} \cdot \mathbf{W})\right)-\gamma^{2} \frac{1}{\hat{R}}(\widehat{\mathbf{R}} \times \mathbf{W})\left(\widehat{\mathbf{R}} \cdot \mathbf{E}_{\mathbf{U}}\right)
\end{aligned}
$$

Although the form of the two-parameter group of field transformations is unclear, it is obvious that the transformations should include only velocity and acceleration and should not depend on $\widehat{\mathbf{R}}$. If the velocity is much smaller than the speed of light, the lag time tends to zero, $\widehat{\mathbf{R}}$ and $\hat{r}$ become close and the transformations may be easier to find. Thus, for an arbitrarily moving charge and a stationary observation point

$$
\begin{gathered}
\mathbf{E}_{\mathrm{s}}=Q \frac{\mathbf{r}}{\mathrm{r}^{3}} \\
\mathbf{B}_{\mathrm{s}}=\mathbf{0} \\
\mathbf{E}_{\mathbf{U}}=\gamma \mathbf{E}_{\mathrm{s}}-\Gamma \mathbf{U}\left(\mathbf{U} \cdot \mathbf{E}_{\mathrm{s}}\right) \\
\mathbf{B}_{\mathbf{U}}=\gamma\left(\mathbf{U} \times \mathbf{E}_{s}\right) \\
\mathbf{E}=\mathbf{E}_{\mathbf{U}}+\gamma^{2}\left(\widehat{\mathbf{R}} \times\left(\mathbf{E}_{\mathbf{U}} \times \mathbf{W}\right)\right) \\
\mathbf{B}=\frac{1}{\hat{R}}(\widehat{\mathbf{R}} \times \mathbf{E}) \\
\widehat{\mathbf{R}}=\hat{\mathbf{r}}+\mathbf{U} \hat{R} \\
\widehat{\mathbf{r}}=\gamma \mathbf{r}-\Gamma \mathbf{U}(\mathbf{U} \cdot \mathbf{r})
\end{gathered}
$$


Consider the same mutual arrangement of the charge and the observation point in space, but now assume that the charge is at rest and the observation point has velocity $\mathbf{V}$ and acceleration $\boldsymbol{\Omega}$. The current time is denoted again by $t$. The vector $\hat{\mathbf{r}}$ is still directed from the point where the charge is located to the observation point. From the point where the charge is located, set a distance equal to the length of the vector $\mathbf{U} \hat{R}$, so that the vector itself is directed from the resulting point to the point where the charge is located. We define the vector $\widehat{\mathbf{R}}$ as the sum of

$$
\widehat{\mathbf{R}}=\mathbf{U} \hat{R}+\hat{\mathbf{r}}
$$

Let us assume that there is a charge moving with velocity $-\mathbf{V}$ and acceleration $-\boldsymbol{\Omega}$ at the obtained point. Then the fields at the observation point will have the form

$$
\begin{gathered}
\mathbf{E}_{\mathbf{s}}=Q \frac{\mathbf{r}}{r^{3}} \\
\mathbf{B}_{\mathbf{s}}=\mathbf{0} \\
\mathbf{E}_{-\mathbf{V}}=\gamma \mathbf{E}_{\mathbf{s}}-\Gamma \mathbf{V}\left(\mathbf{V} \cdot \mathbf{E}_{\mathrm{s}}\right) \\
\mathbf{B}_{-\mathbf{V}}=\gamma\left((-\mathbf{V}) \times \mathbf{E}_{s}\right) \\
\mathbf{E}=\mathbf{E}_{-\mathbf{V}}+\gamma^{2}\left(\widehat{\mathbf{R}} \times\left(\mathbf{E}_{-\mathbf{V}} \times(-\mathbf{\Omega})\right)\right) \\
\mathbf{B}=\frac{1}{\hat{R}}(\widehat{\mathbf{R}} \times \mathbf{E}) \\
\widehat{\mathbf{R}}=\hat{\mathbf{r}}+\mathbf{U} \hat{R} \\
\hat{\mathbf{r}}=\gamma \mathbf{r}-\Gamma \mathbf{V}(\mathbf{V} \cdot \mathbf{r})
\end{gathered}
$$

Here the vector $\mathbf{r}$ is directed from the point where the charge is located to the observation point, and the point where the vectors $\widehat{\mathbf{R}}$ and $\widehat{\mathbf{r}}$ begin is characterized by the fact that the path from it to the point where the charge is located with speed $\mathbf{V}$ takes the same time $\hat{R}$ as the path of light with unit speed to the observation point.

If the observation point moves so that $\mathbf{V}=-\mathbf{U}$ and $\mathbf{\Omega}=-\mathbf{W}$, then the fields in it will be the same when it was at rest and the charge was moving.

If both the charge and the observation point are moving, with $\mathbf{V}=\mathbf{U}$ and $\mathbf{\Omega}=\mathbf{W}$, then they will be stationary relative to each other and the fields will be determined by Coulomb's law. If we find a two-parameter transformation group, we can verify this fact directly. If there is no equality of speeds and accelerations, then two transformations will lead to a third with a speed and acceleration determined by the corresponding formulas for adding speeds and accelerations.

So, we have equations (26) and (27) valid for a uniformly moving observation point. If we put $\mathbf{v}=\mathbf{0}$ in them, then these will be equations valid only for a stationary observation point, that is, Maxwell's equations. The question arises, why not limit ourselves to considering only such equations. Similarly, if we knew the form of the equations valid for an arbitrarily moving observation point, then they would include a parameter that makes sense of acceleration, and if we put it equal to zero, we would come to our equations (26) and (27) for a uniformly moving observation point. So why don't we have enough equations only for a uniformly moving point, and moreover, why can't we confine ourselves to equations for a stationary observation point.

To answer this question, consider a charge that rests in a constant electric field $\mathbf{E}$. The force is acting on him

$$
\mathbf{F}=q \mathbf{E}
$$

If the charge moves uniformly at a constant velocity, then the force will be already

$$
\mathbf{F}=q(\gamma \mathbf{E}-\Gamma \mathbf{U}(\mathbf{U} \cdot \mathbf{E}))
$$


At the point where the charge is located, there is also a magnetic field, but it does not act on the charge.

If the charge also has a non-zero acceleration, then we do not know what force will act on it. We will find out this when we find a two-parameter transformation group. Only in the special case when the field is created by a stationary charge or several stationary charges (so that there are no magnetic fields from them), we can use the formula for the field of an arbitrarily moving charge, as described above.

Thus, in order to correctly determine the force acting on the charge in different situations, we must take into account the nature of the movement of the charge and cannot limit ourselves to the case of its immobility. Similarly, we cannot limit ourselves to equations only for a fixed observation point. In both cases, the issue is solved after finding the necessary transformation group.

We can say that there are no fixed observation points, the slightest movement of the reference frame leads to the movement of the observation point. After all, the observation point is not an abstract concept, there may be a charge in it, and the field acts on a moving charge differently than on a stationary one. Here it is impossible to do with the addition of the Lorentz force, it is also necessary to take into account the multipliers $\gamma \gamma$ and $\Gamma$, so it cannot be said that an electric field plus a magnetic field acts through the Lorentz force. It is correct to say that only the electric field acts, which, firstly, is scaled, and secondly, a contribution from the magnetic field is added to it, and thirdly, there is also an addition from the longitudinal component of the field. In the presence of all these factors at once, we cannot logically formulate how the magnetic field acts on a moving charge by introducing the Lorentz force, this can be done only at low speeds, when $\gamma$ and the longitudinal component can be neglected. And at high speeds, we have to assume that the magnetic field does not act on the charge, but gives a contribution to the electric one.

We will continue to consider the electrodynamic equations, realizing that they are valid only for a uniformly moving observation point. If its movement is arbitrary, they will become more complicated, but the physical manifestations of the already known members will not go away and with their help you can make predictions for the results of experiments.

So, at the moment we have the equations

$$
\begin{gathered}
\operatorname{rot}(\gamma(\mathbf{E}-(\mathbf{v} \times \mathbf{B}))-\Gamma \mathbf{v}(\mathbf{v} \cdot \mathbf{E}))=-\frac{\partial}{\partial t}(\gamma(\mathbf{B}+(\mathbf{v} \times \mathbf{E}))-\Gamma \mathbf{v}(\mathbf{v} \cdot \mathbf{B})) \\
\operatorname{rot}(\gamma(\mathbf{B}+(\mathbf{v} \times \mathbf{E}))-\Gamma \mathbf{v}(\mathbf{v} \cdot \mathbf{B}))=\frac{\partial}{\partial t}(\gamma(\mathbf{E}-(\mathbf{v} \times \mathbf{B}))-\Gamma \mathbf{v}(\mathbf{v} \cdot \mathbf{E}))+4 \pi \hat{\mathbf{\jmath}} \\
\operatorname{div}(\gamma(\mathbf{E}-(\mathbf{v} \times \mathbf{B}))-\Gamma \mathbf{v}(\mathbf{v} \cdot \mathbf{E}))=4 \pi \hat{\rho} \\
\operatorname{div}(\gamma(\mathbf{B}+(\mathbf{v} \times \mathbf{E}))-\Gamma \mathbf{v}(\mathbf{v} \cdot \mathbf{B}))=0 \\
\gamma=\frac{1}{\sqrt{1-v^{2}}} \quad \Gamma=\frac{\gamma-1}{v^{2}}
\end{gathered}
$$

Now

$$
\begin{aligned}
\widehat{\mathbf{E}} & =\gamma(\mathbf{E}-(\mathbf{v} \times \mathbf{B}))-\Gamma \mathbf{v}(\mathbf{v} \cdot \mathbf{E}) \\
\widehat{\mathbf{B}} & =\gamma(\mathbf{B}+(\mathbf{v} \times \mathbf{E}))-\Gamma \mathbf{v}(\mathbf{v} \cdot \mathbf{B})
\end{aligned}
$$

The inverse transformations have the same form with a reversed sign of velocity.

Again, all the reasoning given from the beginning of the article remains valid, in particular, the plane and spherical wave are solutions of the equations in the absence of currents and 
charges, since the fields of the plane wave and spherical wave have only transverse components. This is a good argument in favor of the truth of the equations in terms of transverse fields. As for the longitudinal fields, the truth of the equations taking them into account can be checked in the experiment. At the end of the article, new types of alternators of alternating and direct current are proposed. In case of their performance the correctness of equations of electrodynamics in form (30), (31), (32) and (33) will be confirmed in case of uniform motion of observation point.

From the equations for the rotors and from the continuity equation for the current follow the equations for divergences and the expression for the bias current $\frac{\partial}{\partial t}\left(\frac{\mathbf{E}-(\mathbf{v} \times \mathbf{B})}{\sqrt{1-v^{2}}}-\Gamma \mathbf{v}(\mathbf{v} \cdot \mathbf{E})\right)$. The wave equations with sources for vector and scalar potentials are still true,

$$
\begin{gathered}
\nabla^{2} \widehat{\mathbf{A}}-\frac{1}{c^{2}} \frac{\partial^{2} \widehat{\mathbf{A}}}{\partial t^{2}}=-4 \pi \hat{\mathbf{\jmath}} \\
\nabla^{2} \hat{\varphi}-\frac{1}{c^{2}} \frac{\partial^{2} \hat{\varphi}}{\partial t^{2}}=-4 \pi \hat{\rho} \\
\operatorname{div} \widehat{\mathbf{A}}=-\frac{\partial \hat{\varphi}}{\partial t}
\end{gathered}
$$

and the fields are expressed through them by the formulas

$$
\begin{gathered}
\widehat{\mathbf{B}}=\gamma(\mathbf{B}+(\mathbf{v} \times \mathbf{E}))-\Gamma \mathbf{v}(\mathbf{v} \cdot \mathbf{B})=\operatorname{rot} \widehat{\mathbf{A}} \\
\widehat{\mathbf{E}}=\gamma(\mathbf{E}-(\mathbf{v} \times \mathbf{B}))-\Gamma \mathbf{v}(\mathbf{v} \cdot \mathbf{E})=-\operatorname{grad} \hat{\varphi}-\frac{\partial \widehat{\mathbf{A}}}{\partial t}
\end{gathered}
$$

It is possible not to find the fields by means of field transformations, but to first transform the potentials by the formulas ([3], Chapter 7)

$$
\begin{gathered}
\varphi=\gamma(\hat{\varphi}-(\mathbf{v} \cdot \widehat{\mathbf{A}})) \\
\mathbf{A}=\widehat{\mathbf{A}}-\gamma \mathbf{v} \hat{\varphi}+\Gamma \mathbf{v}(\mathbf{v} \cdot \widehat{\mathbf{A}}) \\
\hat{\varphi}=\gamma(\varphi+(\mathbf{v} \cdot \mathbf{A})) \\
\widehat{\mathbf{A}}=\mathbf{A}+\gamma \mathbf{v} \varphi+\Gamma \mathbf{v}(\mathbf{v} \cdot \mathbf{A})
\end{gathered}
$$

and obtain from them the fields

$$
\begin{gathered}
\mathbf{B}=\operatorname{rot} \mathbf{A} \\
\mathbf{E}=-\operatorname{grad} \varphi-\frac{\partial \mathbf{A}}{\partial t}
\end{gathered}
$$

or one can immediately solve the wave equations

$$
\begin{gathered}
\nabla^{2} \mathbf{A}-\frac{1}{c^{2}} \frac{\partial^{2} \mathbf{A}}{\partial t^{2}}=-4 \pi \mathbf{j} \\
\nabla^{2} \varphi-\frac{1}{c^{2}} \frac{\partial^{2} \varphi}{\partial t^{2}}=-4 \pi \rho \\
\operatorname{div} \mathbf{A}=-\frac{1}{c^{2}} \frac{\partial \varphi}{\partial t}
\end{gathered}
$$

or one can immediately solve the wave equations

$$
\begin{gathered}
\rho=\gamma(\hat{\rho}-(\mathbf{v} \cdot \hat{\mathbf{j}})) \\
\mathbf{j}=\hat{\mathbf{j}}-\gamma \mathbf{v} \hat{\rho}+\Gamma \mathbf{v}(\mathbf{v} \cdot \hat{\mathbf{j}}) \\
\hat{\rho}=\gamma(\rho+(\mathbf{v} \cdot \mathbf{j})) \\
\hat{\mathbf{j}}=\mathbf{j}+\gamma \mathbf{v} \rho+\Gamma \mathbf{v}(\mathbf{v} \cdot \mathbf{j})
\end{gathered}
$$

Although the transformations of electric and magnetic fields coincide in appearance with the Lorentz transformations for fields during the transition from one inertial reference system to another (see, for example, [3], Chapter 7), we consider them as an integral part of the equations 
of electrodynamics. Only in this form do the equations have as solutions plane and spherical waves, as well as the fields of a uniformly moving charge. In this case, $v$ in them is a free parameter, different values of which allow specific configurations of fields to exist. If the charge moves arbitrarily, its fields depend also on acceleration, so that the parameter $\mathrm{v}$ alone is not enough to ensure that the equations at some point are fulfilled, we must include in the equations some other parameter with the dimension of acceleration. If the necessary expression is found, the equations will become universal, since they will satisfy the fields of any combination of arbitrarily moving charges, and in formula (6) for EMF there will be terms that depend on acceleration, the effect of which can be found in the experiment.

Equations (30) and (31) can be considered as two experimentally based electrodynamic equations for rotors, from which two more equations for divergences are derived using the current continuity equation. They do not require references to the theory of relativity in terms of coordinate and time transformations. The equations of electrodynamics and the field transformations built into them can be considered primary. They describe electrodynamics in the same frame of reference, and only fields, potentials and currents, but not space and time, are subjected to transformations.

Let us consider the Aharonov-Bohm effect, in which the run-up of the quantummechanical phase of a charged particle along a closed loop, which can de expressed by the Stokes' theorem through the surface integral of the magnetic field, that is, through the magnetic flux through the surface stretched on the loop

$$
\oint_{L} \mathbf{A} \cdot d \mathbf{l}=\int_{S} \operatorname{rot} \mathbf{A} \cdot d \mathbf{s}=\int_{S} \mathbf{B} \cdot d \mathbf{s}
$$

In light of the above, it is logical to assume that when calculating the phase overrun it is necessary to take into account not vector $\mathbf{A}$, but vector $\widehat{\mathbf{A}}$, that is, we should write

$$
\oint_{L}(\mathbf{A}+\gamma \mathbf{v} \varphi+\Gamma \mathbf{v}(\mathbf{v} \cdot \mathbf{A})) \cdot d \mathbf{l}=\int_{S} \operatorname{rot} \widehat{\mathbf{A}} \cdot d \mathbf{s}=\int_{S}(\gamma(\mathbf{B}+(\mathbf{v} \times \mathbf{E}))-\Gamma \mathbf{v}(\mathbf{v} \cdot \mathbf{B})) \cdot d \mathbf{s}
$$

\section{Equation of motion}

The above reasoning contradicts to the fact that relativistic equation of motion of a charge in electromagnetic field is considered to be known and has a form ([2], Chapter 26, Paragraph 4, page 280)

$$
\frac{d}{d t}\left(\frac{m \mathbf{U}}{\sqrt{1-U^{2}}}\right)=\mathbf{F}=q(\mathbf{E}+(\mathbf{U} \times \mathbf{B}))
$$

where $m$ is the mass of the particle carrying the charge, $\mathbf{F}$ is the force acting on the particle. In addition, it is true for the derivative of the kinetic energy

$$
\frac{d}{d t}\left(\frac{m}{\sqrt{1-U^{2}}}\right)=q(\mathbf{U} \cdot \mathbf{E})
$$

Here the charge is moving accelerated and if the formula is correct, then it turns out that we do know the force acting on the accelerated moving charge from the electric field and even a combination of electric and magnetic fields. Although in [2] it is not explicitly stated, but it is obvious that there by $\mathbf{E}$ and $\mathbf{B}$ we mean the fields at a stationary point of observation.

We have assumed above that the force acting on a uniformly moving charge is 


$$
\mathbf{F}=q \mathbf{E}_{\mathbf{U}}=q \gamma(\mathbf{E}+(\mathbf{U} \times \mathbf{B}))-q \Gamma \mathbf{U}(\mathbf{U} \cdot \mathbf{E})
$$

We could say that we should substitute it in the right part of the equation for the derivative of momentum, but this would be incorrect because it is a force for the case of uniform motion, and if momentum changes, the motion is obviously non-uniform, and for such a case we do not know the formula for the field and force. Nevertheless, it seems that substituting such a force into the equation of motion gives a result closer to reality

$$
\begin{gathered}
\frac{d \mathbf{P}}{d t}=q \gamma(\mathbf{E}+(\mathbf{U} \times \mathbf{B}))-q \Gamma \mathbf{U}(\mathbf{U} \cdot \mathbf{E}) \\
\frac{d \mathcal{E}}{d t}=q(\mathbf{U} \cdot(\gamma(\mathbf{E}+(\mathbf{U} \times \mathbf{B}))-\Gamma \mathbf{U}(\mathbf{U} \cdot \mathbf{E})))=q \gamma(\mathbf{U} \cdot \mathbf{E})-q \Gamma U^{2}(\mathbf{U} \cdot \mathbf{E}) \\
=q \gamma(\mathbf{U} \cdot \mathbf{E})-q(\gamma-1)(\mathbf{U} \cdot \mathbf{E})=q(\mathbf{U} \cdot \mathbf{E})
\end{gathered}
$$

здесь использованы обозначения для релятивистского импульса и кинетической энергии

$$
\mathbf{P} \equiv \frac{m \mathbf{U}}{\sqrt{1-U^{2}}} \quad \mathcal{E} \equiv \frac{m}{\sqrt{1-U^{2}}}
$$

Note that only the expression for the derivative of momentum has changed, while the expression for the derivative of kinetic energy remains the same. It turns out that in both cases all the work of the electric field goes to change the kinetic energy of the particle and nothing is spent on radiation. But this contradicts the fact that the particle moves accelerated and must radiate energy.

If we find the correct formula for the field at an arbitrarily moving point of observation, we substitute it in the right part of the equation of motion and there appears the acceleration of the particle.

For a particular case of motion of a particle in the field of a stationary charge $Q$ we know the expression for the electric field (here $\mathbf{a} \equiv \frac{\partial \mathbf{U}}{\partial t}$ is the acceleration of the particle)

$$
\begin{gathered}
\mathbf{E}=\mathbf{E}_{\mathbf{U}}-\gamma^{2}\left(\widehat{\mathbf{R}} \times\left(\mathbf{E}_{\mathbf{U}} \times \mathbf{a}\right)\right) \\
\mathbf{E}_{\mathbf{U}}=\gamma \mathbf{E}_{\mathbf{s}}-\Gamma \mathbf{U}\left(\mathbf{U} \cdot \mathbf{E}_{\mathrm{s}}\right) \\
\mathbf{E}_{\mathbf{s}}=Q \frac{\mathbf{r}}{r^{3}} \\
\widehat{\mathbf{R}}=\hat{\mathbf{r}}+\mathbf{U} \hat{R} \\
\hat{\mathbf{r}}=\gamma r-\Gamma \mathbf{U}(\mathbf{U} \cdot \mathbf{r})
\end{gathered}
$$

For the kinetic energy derivative, we obtain

$$
\begin{aligned}
\frac{d \mathcal{E}}{d t}=q(\mathbf{U} \cdot \mathbf{E}) & =q\left(\mathbf{U} \cdot \mathbf{E}_{\mathbf{U}}\right)-q \gamma^{2}\left(\mathbf{U} \cdot\left(\widehat{\mathbf{R}} \times\left(\mathbf{E}_{\mathbf{U}} \times \mathbf{a}\right)\right)\right) \\
& =q\left(\mathbf{U} \cdot\left(\gamma \mathbf{E}_{\mathbf{s}}-\Gamma \mathbf{U}\left(\mathbf{U} \cdot \mathbf{E}_{\mathbf{s}}\right)\right)\right)-q \gamma^{2}\left(\mathbf{U} \cdot\left(\widehat{\mathbf{R}} \times\left(\mathbf{E}_{\mathbf{U}} \times \mathbf{a}\right)\right)\right) \\
& =q \gamma\left(\mathbf{U} \cdot \mathbf{E}_{\mathbf{s}}\right)-q \Gamma U^{2}\left(\mathbf{U} \cdot \mathbf{E}_{\mathrm{s}}\right)-q \gamma^{2}\left(\mathbf{U} \cdot\left(\widehat{\mathbf{R}} \times\left(\mathbf{E}_{\mathbf{U}} \times \mathbf{a}\right)\right)\right) \\
& =q \gamma\left(\mathbf{U} \cdot \mathbf{E}_{\mathrm{s}}\right)-q(\gamma-1)\left(\mathbf{U} \cdot \mathbf{E}_{\mathrm{s}}\right)-q \gamma^{2}\left(\mathbf{U} \cdot\left(\widehat{\mathbf{R}} \times\left(\mathbf{E}_{\mathbf{U}} \times \mathbf{a}\right)\right)\right) \\
& =q\left(\mathbf{U} \cdot \mathbf{E}_{\mathrm{s}}\right)-q \gamma^{2}\left(\mathbf{U} \cdot\left(\widehat{\mathbf{R}} \times\left(\mathbf{E}_{\mathbf{U}} \times \mathbf{a}\right)\right)\right)
\end{aligned}
$$

Now we can see that the work of the electric field is not only spent on changing the kinetic energy, but also part of it goes to radiation. This formula does not yet take into account the change in potential energy of the system of charges $q$ and $Q$. Let's find the change in total energy 


$$
\begin{aligned}
\frac{d}{d t}\left(\frac{m}{\sqrt{1-U^{2}}}+\right. & \left.\frac{q Q}{r}\right)=q\left(\mathbf{U} \cdot \mathbf{E}_{\mathbf{S}}\right)-q \gamma^{2}\left(\mathbf{U} \cdot\left(\widehat{\mathbf{R}} \times\left(\mathbf{E}_{\mathbf{U}} \times \mathbf{a}\right)\right)\right)-\frac{q Q}{r^{2}} \frac{d r}{d t} \\
& =q\left(\mathbf{U} \cdot Q \frac{\mathbf{r}}{r^{3}}\right)-q \gamma^{2}\left(\mathbf{U} \cdot\left(\widehat{\mathbf{R}} \times\left(\mathbf{E}_{\mathbf{U}} \times \mathbf{a}\right)\right)\right)-\frac{q Q}{r^{2}} \frac{d r}{d t} \\
& =q\left(\mathbf{U} \cdot Q \frac{\mathbf{r}}{r^{3}}\right)-q \gamma^{2}\left(\mathbf{U} \cdot\left(\widehat{\mathbf{R}} \times\left(\mathbf{E}_{\mathbf{U}} \times \mathbf{a}\right)\right)\right)-\frac{q Q}{r^{3}}(\mathbf{U} \cdot \mathbf{r}) \\
& =-q \gamma^{2}\left(\mathbf{U} \cdot\left(\widehat{\mathbf{R}} \times\left(\mathbf{E}_{\mathbf{U}} \times \mathbf{a}\right)\right)\right)=-q \gamma^{2}\left(\mathbf{U} \cdot\left(\mathbf{E}_{\mathbf{U}}(\widehat{\mathbf{R}} \cdot \mathbf{a})+\mathbf{a}\left(\widehat{\mathbf{R}} \cdot \mathbf{E}_{\mathbf{U}}\right)\right)\right) \\
& =-q \gamma^{2}\left(\mathbf{U} \cdot \mathbf{E}_{\mathbf{U}}\right)(\widehat{\mathbf{R}} \cdot \mathbf{a})+q \gamma^{2}(\mathbf{U} \cdot \mathbf{a})\left(\widehat{\mathbf{R}} \cdot \mathbf{E}_{\mathbf{U}}\right)= \\
& =-q \gamma^{2}\left(\mathbf{U} \cdot \mathbf{E}_{\mathbf{S}}\right)(\widehat{\mathbf{R}} \cdot \mathbf{a})+q \gamma^{2}(\mathbf{U} \cdot \mathbf{a})\left(\widehat{\mathbf{R}} \cdot \mathbf{E}_{\mathbf{U}}\right)
\end{aligned}
$$

here it is taken into account that

$$
\begin{gathered}
\frac{d r}{d t}=\frac{1}{r} r \frac{d r}{d t}=\frac{1}{2 r} \frac{d r^{2}}{d t}=\frac{1}{2 r} \frac{r_{x}^{2}+r_{y}^{2}+r_{z}^{2}}{d t}=\frac{1}{r}\left(r_{x} \frac{d r_{x}}{d t}+r_{y} \frac{d r_{y}}{d t}+r_{z} \frac{d r_{z}}{d t}\right) \\
=\frac{1}{r}\left(r_{x} U_{x}+r_{y} U_{y}+r_{z} U_{z}\right)=\frac{1}{r}(\mathbf{U} \cdot \mathbf{r}) \\
\left(\mathbf{U} \cdot \mathbf{E}_{\mathbf{U}}\right)=\left(\mathbf{U} \cdot\left(\gamma \mathbf{E}_{\mathrm{s}}-\Gamma \mathbf{U}\left(\mathbf{U} \cdot \mathbf{E}_{\mathrm{s}}\right)\right)\right)=\gamma\left(\mathbf{U} \cdot \mathbf{E}_{\mathrm{s}}\right)-\Gamma U^{2}\left(\mathbf{U} \cdot \mathbf{E}_{\mathrm{s}}\right)=\left(\mathbf{U} \cdot \mathbf{E}_{\mathrm{s}}\right)
\end{gathered}
$$

Thus, the total energy decreases due to radiation as the particle accelerates.

For the momentum derivative we have

$$
\frac{d \mathbf{P}}{d t}=q \mathbf{E}_{\mathbf{U}}-\gamma^{2} q\left(\widehat{\mathbf{R}} \times\left(\mathbf{E}_{\mathbf{U}} \times \mathbf{a}\right)\right)
$$

Учитывая соотношение, которое будет проверено далее в статье

$$
\frac{d \mathbf{P}}{d t}=\frac{4 m}{\left(\sqrt{1-U^{2}}\right)^{3}}(\mathbf{a}+(\mathbf{U} \times(\mathbf{U} \times \mathbf{a})))
$$

можем записать

$$
4 m \gamma^{3}(\mathbf{a}+(\mathbf{U} \times(\mathbf{U} \times \mathbf{a})))=q \mathbf{E}_{\mathbf{U}}-\gamma^{2} q\left(\widehat{\mathbf{R}} \times\left(\mathbf{E}_{\mathbf{U}} \times \mathbf{a}\right)\right)
$$

If the charge $Q$ also moves, there will be a magnetic field at the stationary point of observation as well. For a moving charge $Q$, we do not yet know the expression for the electric field at an arbitrarily moving point of observation.

It is known that the Lagrange function for a charged particle in an electromagnetic field is

$$
L=-m \sqrt{1-U^{2}}-q \varphi+q(\mathbf{A} \cdot \mathbf{U})
$$

The motion of the particle is described by the Lagrange equation

Here

$$
\begin{aligned}
& \frac{d}{d t}\left(\frac{\partial L}{\partial \mathbf{U}}\right)=\frac{\partial L}{\partial \mathbf{r}} \equiv \boldsymbol{\nabla} L \\
& \frac{\partial L}{\partial \mathbf{U}}=\frac{m \mathbf{U}}{\sqrt{1-U^{2}}}+q \mathbf{A}
\end{aligned}
$$

$$
\begin{gathered}
\boldsymbol{\nabla} \equiv \mathbf{i} \frac{\partial}{\partial x}+\mathbf{j} \frac{\partial}{\partial y}+\mathbf{k} \frac{\partial}{\partial z} \\
\frac{\partial L}{\partial \mathbf{r}} \equiv \boldsymbol{\nabla} L \equiv L \boldsymbol{\nabla} \equiv \mathbf{i} \frac{\partial L}{\partial x}+\mathbf{j} \frac{\partial L}{\partial y}+\mathbf{k} \frac{\partial L}{\partial z} \equiv\left(\frac{\partial L}{\partial x}, \frac{\partial L}{\partial y}, \frac{\partial L}{\partial z}\right)^{T}
\end{gathered}
$$

In [5] (Sec. 3, para. 17, p. 72) the formula is used

$$
\operatorname{grad}(\mathbf{A} \cdot \mathbf{U})=(\mathbf{A} \cdot \boldsymbol{\nabla}) \mathbf{U}+(\mathbf{U} \cdot \boldsymbol{\nabla}) \mathbf{A}+(\mathbf{U} \times \operatorname{rot} \mathbf{A})+(\mathbf{A} \times \operatorname{rot} \mathbf{U})
$$

and states that the coordinate derivatives are taken at a constant $\mathbf{U}$, which gives

$$
\operatorname{grad}(\mathbf{A} \cdot \mathbf{U})=(\mathbf{U} \cdot \boldsymbol{\nabla}) \mathbf{A}+(\mathbf{U} \times \operatorname{rot} \mathbf{A})
$$


The constancy of velocity is a strong assumption that simplifies the calculations and allows the definition of the Lorentz force to be introduced $q(\mathbf{E}+(\mathbf{U} \times \mathbf{B}))$. But such a representation cannot be accurate, since the force causes acceleration and variability of velocity.

If we consider the velocity as constant, we must immediately recognize that in the Lagrange equation we take only the partial derivative on time from the relativistic momentum, while we take the full derivative on time from the other part of the generalized momentum - from the vector potential. If we take as the force acting on the particle the partial derivative of the relativistic momentum, taking the full derivative from the vector potential and considering the velocity as independent of coordinates, then we obtain the Lorentz force. If we take as the force the full derivative of the relativistic momentum, then in other terms it is necessary to take into account the dependence of velocity on coordinates, and then the force will not be equal to the Lorentz force and will depend on velocity and acceleration.

Further we will accept the definition of the force by the full derivative of the relativistic momentum and we will leave the equation of motion in the uncut form for the varying velocity and use more correct formula for the gradient of the scalar product

$$
\boldsymbol{\nabla}(\mathbf{A} \cdot \mathbf{U})=(\mathbf{A} \boldsymbol{\nabla}) \mathbf{U}+(\mathbf{U} \boldsymbol{\nabla}) \mathbf{A}+(\mathbf{U} \times \operatorname{rot} \mathbf{A})+(\mathbf{A} \times \operatorname{rot} \mathbf{U})
$$

where $\mathbf{A}$ and $\mathbf{U}$ are vector columns, and the matrices $(\mathbf{A} \boldsymbol{\nabla})$ and $(\mathbf{U} \boldsymbol{\nabla})$ are defined as follows

$$
\mathbf{A} \boldsymbol{\nabla} \equiv\left[\begin{array}{lll}
\frac{\partial A_{x}}{\partial x} & \frac{\partial A_{x}}{\partial y} & \frac{\partial A_{x}}{\partial z} \\
\frac{\partial A_{y}}{\partial x} & \frac{\partial A_{y}}{\partial y} & \frac{\partial A_{y}}{\partial z} \\
\frac{\partial A_{z}}{\partial x} & \frac{\partial A_{z}}{\partial y} & \frac{\partial A_{z}}{\partial z}
\end{array}\right] \quad \mathbf{U} \equiv\left[\begin{array}{lll}
\frac{\partial U_{x}}{\partial x} & \frac{\partial U_{x}}{\partial y} & \frac{\partial U_{x}}{\partial z} \\
\frac{\partial U_{y}}{\partial x} & \frac{\partial U_{y}}{\partial y} & \frac{\partial U_{y}}{\partial z} \\
\frac{\partial U_{z}}{\partial x} & \frac{\partial U_{z}}{\partial y} & \frac{\partial U_{z}}{\partial z}
\end{array}\right]
$$

Given the expression for the total differential of the vector potential

we obtain

$$
\frac{d \mathbf{A}}{d t}=\frac{\partial \mathbf{A}}{\partial t}+(\mathbf{A} \boldsymbol{\nabla}) \mathbf{U}
$$

$$
\begin{aligned}
\begin{aligned}
\frac{d}{d t}\left(\frac{m \mathbf{U}}{\sqrt{1-U^{2}}}+\right. & q \mathbf{A})=\boldsymbol{\nabla} L=-m \boldsymbol{\nabla}\left(\sqrt{1-U^{2}}\right)+q \boldsymbol{\nabla}(\mathbf{A} \cdot \mathbf{U})-q \boldsymbol{\nabla} \varphi \\
& =-m \boldsymbol{\nabla}\left(\sqrt{1-U^{2}}\right)+q(\mathbf{A} \boldsymbol{\nabla}) \mathbf{U}+q(\mathbf{U} \boldsymbol{\nabla}) \mathbf{A}+q(\mathbf{U} \times \operatorname{rot} \mathbf{A})+q(\mathbf{A} \times \operatorname{rot} \mathbf{U}) \\
& -q \boldsymbol{\nabla} \varphi
\end{aligned} \\
\frac{d \mathbf{P}}{d t}=-m \boldsymbol{\nabla}\left(\sqrt{1-U^{2}}\right)-q \frac{\partial \mathbf{A}}{\partial t}-q \boldsymbol{\nabla} \varphi+q(\mathbf{U} \times \operatorname{rot} \mathbf{A})+q(\mathbf{U} \boldsymbol{\nabla}) \mathbf{A}+q(\mathbf{A} \times \operatorname{rot} \mathbf{U})
\end{aligned}
$$

Let's write down the ratios

$$
\begin{array}{ll}
\mathbf{P}=\varepsilon \mathbf{U} & \frac{d \mathbf{P}}{d t}=\mathbf{U} \frac{d \varepsilon}{d t}+\varepsilon \frac{d \mathbf{U}}{d t} \\
\frac{d \mathcal{E}}{d t}=\frac{\partial \mathcal{E}}{\partial t}+(\boldsymbol{\nabla} E) \mathbf{U} & \frac{d \mathbf{P}}{d t}=\frac{\partial \mathbf{P}}{\partial t}+(\mathbf{P} \boldsymbol{\nabla}) \mathbf{U} \\
\frac{d \mathbf{A}}{d t}=\frac{\partial \mathbf{A}}{\partial t}+(\mathbf{A} \boldsymbol{\nabla}) \mathbf{U} & \frac{d \mathbf{U}}{d t}=\frac{\partial \mathbf{U}}{\partial t}+(\mathbf{U \nabla}) \mathbf{U}
\end{array}
$$

Then the Lagrange equation will take the form

$$
\frac{d \mathbf{P}}{d t}+q \frac{d \mathbf{A}}{d t}=-m \boldsymbol{\nabla}\left(\sqrt{1-U^{2}}\right)-q \frac{\partial \mathbf{A}}{\partial t}-q \boldsymbol{\nabla} \varphi+q(\mathbf{U} \times \operatorname{rot} \mathbf{A})+q(\mathbf{U} \boldsymbol{\nabla}) \mathbf{A}+q(\mathbf{A} \times \operatorname{rot} \mathbf{U})
$$


$\frac{d \mathbf{P}}{d t}+m \boldsymbol{\nabla}\left(\sqrt{1-U^{2}}\right)=-q \frac{\partial \mathbf{A}}{\partial t}-q \boldsymbol{\nabla} \varphi+q(\mathbf{U} \times \operatorname{rot} \mathbf{A})+q(\mathbf{U} \boldsymbol{\nabla}) \mathbf{A}+q(\mathbf{A} \times \operatorname{rot} \mathbf{U})$

On the one hand, the total derivative of the kinetic energy can be found directly

$$
\begin{gathered}
\frac{d \varepsilon}{d t}=\frac{d}{d t}\left(\frac{m}{\sqrt{1-U^{2}}}\right)=-\frac{1}{2} \frac{m}{\left(\sqrt{1-U^{2}}\right)^{3}} \frac{d}{d t}\left(1-U^{2}\right)=\frac{1}{2} \frac{m}{\left(\sqrt{1-U^{2}}\right)^{3}} \frac{d U^{2}}{d t} \\
=\frac{m}{\left(\sqrt{1-U^{2}}\right)^{3}}\left(\mathbf{U} \cdot \frac{d \mathbf{U}}{d t}\right)
\end{gathered}
$$

On the other hand, it is possible to find an expression

$$
\begin{aligned}
\left(\mathbf{U} \cdot \frac{d \mathbf{P}}{d t}\right)=(\mathbf{U} & \left.\cdot\left(\mathbf{U} \frac{d \mathcal{E}}{d t}+\varepsilon \frac{d \mathbf{U}}{d t}\right)\right)=U^{2} \frac{d \mathcal{E}}{d t}+\varepsilon\left(\mathbf{U} \cdot \frac{d \mathbf{U}}{d t}\right) \\
= & U^{2} \frac{m}{\left(\sqrt{1-U^{2}}\right)^{3}}\left(\mathbf{U} \cdot \frac{d \mathbf{U}}{d t}\right)+\frac{m}{\sqrt{1-U^{2}}}\left(\mathbf{U} \cdot \frac{d \mathbf{U}}{d t}\right) \\
= & \left(\mathbf{U} \cdot \frac{d \mathbf{U}}{d t}\right)\left(U^{2} \frac{m}{\left(\sqrt{1-U^{2}}\right)^{3}}+\frac{m}{\sqrt{1-U^{2}}}\right)=\left(\mathbf{U} \cdot \frac{d \mathbf{U}}{d t}\right)\left(\frac{m U^{2}+m-m U^{2}}{\left(\sqrt{1-U^{2}}\right)^{3}}\right) \\
= & \frac{m}{\left(\sqrt{1-U^{2}}\right)^{3}}\left(\mathbf{U} \cdot \frac{d \mathbf{U}}{d t}\right)
\end{aligned}
$$

Thus

$$
\frac{d \mathcal{E}}{d t}=\left(\mathbf{U} \cdot \frac{d \mathbf{P}}{d t}\right)
$$

$$
\begin{aligned}
& (\mathbf{U \nabla}) \mathbf{U}=\left(\left(U_{x} \frac{\partial U_{x}}{\partial x}+U_{y} \frac{\partial U_{x}}{\partial y}+U_{z} \frac{\partial U_{x}}{\partial z}\right),\left(U_{x} \frac{\partial U_{y}}{\partial x}+U_{y} \frac{\partial U_{y}}{\partial y}+U_{z} \frac{\partial U_{y}}{\partial z}\right),\left(U_{x} \frac{\partial U_{z}}{\partial x}+U_{y} \frac{\partial U_{z}}{\partial y}\right.\right. \\
& \left.\left.+U_{z} \frac{\partial U_{z}}{\partial z}\right)\right) \\
& =\left(\left(\frac{\partial x}{\partial t} \frac{\partial U_{x}}{\partial x}+\frac{\partial y}{\partial t} \frac{\partial U_{x}}{\partial y}+\frac{\partial z}{\partial t} \frac{\partial U_{x}}{\partial z}\right),\left(\frac{\partial x}{\partial t} \frac{\partial U_{y}}{\partial x}+\frac{\partial y}{\partial t} \frac{\partial U_{y}}{\partial y}+\frac{\partial z}{\partial t} \frac{\partial U_{y}}{\partial z}\right),\left(\frac{\partial x}{\partial t} \frac{\partial U_{z}}{\partial x}\right.\right. \\
& \left.\left.+\frac{\partial y}{\partial t} \frac{\partial U_{z}}{\partial y}+\frac{\partial z}{\partial t} \frac{\partial U_{z}}{\partial z}\right)\right) \\
& =\left(\left(\frac{\partial U_{x}}{\partial t}+\frac{\partial U_{x}}{\partial t}+\frac{\partial U_{x}}{\partial t}\right),\left(\frac{\partial U_{y}}{\partial t}+\frac{\partial U_{y}}{\partial t}+\frac{\partial U_{y}}{\partial t}\right),\left(\frac{\partial U_{z}}{\partial t}+\frac{\partial U_{z}}{\partial t}+\frac{\partial U_{z}}{\partial t}\right)\right) \\
& =3\left(\frac{\partial U_{x}}{\partial t}, \frac{\partial U_{y}}{\partial t}, \frac{\partial U_{z}}{\partial t}\right)=3 \frac{\partial \mathbf{U}}{\partial t} \\
& \frac{d \mathbf{U}}{d t}=4 \frac{\partial \mathbf{U}}{\partial t} \\
& \frac{d \mathcal{E}}{d t}=\frac{4 m}{\left(\sqrt{1-U^{2}}\right)^{3}}\left(\mathbf{U} \cdot \frac{\partial \mathbf{U}}{\partial t}\right)=\frac{4 m}{\left(\sqrt{1-U^{2}}\right)^{3}}(\mathbf{U} \cdot \mathbf{a}) \\
& \left(\frac{1}{2} \frac{\partial U^{2}}{\partial t}\right)=\left(\mathbf{U} \cdot \frac{\partial \mathbf{U}}{\partial t}\right)=(\mathbf{U} \cdot \mathbf{a})
\end{aligned}
$$


Knowing the total derivative of energy, we find the total derivative of momentum, that is, the force.

$$
\begin{array}{r}
\frac{d \mathbf{P}}{d t}=\mathbf{U} \frac{4 m}{\left(\sqrt{1-U^{2}}\right)^{3}}(\mathbf{U} \cdot \mathbf{a})+\varepsilon \frac{d \mathbf{U}}{d t}=\mathbf{U} \frac{4 m}{\left(\sqrt{1-U^{2}}\right)^{3}}(\mathbf{U} \cdot \mathbf{a})+\frac{4 m}{\sqrt{1-U^{2}}} \mathbf{a}= \\
=\frac{4 m}{\sqrt{1-U^{2}}}\left(\frac{\mathbf{U}(\mathbf{U} \cdot \mathbf{a})}{1-U^{2}}+\mathbf{a}\right)=\frac{4 m}{\left(\sqrt{1-U^{2}}\right)^{3}}\left(\mathbf{U}(\mathbf{U} \cdot \mathbf{a})+\mathbf{a}-U^{2} \mathbf{a}\right) \\
=\frac{4 m}{\left(\sqrt{1-U^{2}}\right)^{3}}(\mathbf{a}+(\mathbf{U} \times(\mathbf{U} \times \mathbf{a})))
\end{array}
$$

Let's substitute this expression into the Lagrange equation

$$
\begin{aligned}
\frac{4 m}{\left(\sqrt{1-U^{2}}\right)^{3}}(\mathbf{a} & +(\mathbf{U} \times(\mathbf{U} \times \mathbf{a})))+m \boldsymbol{\nabla}\left(\sqrt{1-U^{2}}\right) \\
= & -q \frac{\partial \mathbf{A}}{\partial t}-q \boldsymbol{\nabla} \varphi+q(\mathbf{U} \times \operatorname{rot} \mathbf{A})+q(\mathbf{U} \boldsymbol{\nabla}) \mathbf{A}+q(\mathbf{A} \times \operatorname{rot} \mathbf{U})
\end{aligned}
$$

Let's find the scalar product of both parts with velocity, taking into account the expression

$$
\begin{gathered}
\left(\mathbf{U} \cdot \boldsymbol{\nabla}\left(\sqrt{1-U^{2}}\right)\right)=\left(U_{x} \frac{\partial\left(\sqrt{1-U^{2}}\right)}{\partial x}+U_{y} \frac{\partial\left(\sqrt{1-U^{2}}\right)}{\partial y}+U_{z} \frac{\partial\left(\sqrt{1-U^{2}}\right)}{\partial z}\right) \\
\frac{\partial\left(\sqrt{1-U^{2}}\right)}{\partial x}=-\frac{1 / 2}{\sqrt{1-U^{2}}} \frac{\partial U^{2}}{\partial x}=-\frac{1}{\sqrt{1-U^{2}}}\left(U_{x} \frac{\partial U_{x}}{\partial x}+U_{y} \frac{\partial U_{y}}{\partial x}+U_{z} \frac{\partial U_{z}}{\partial x}\right) \\
\frac{\partial U^{2}}{\partial x}=\frac{\partial}{\partial x}\left(U_{x}^{2}+U_{y}^{2}+U_{z}^{2}\right)=2 U_{x} \frac{\partial U_{x}}{\partial x}+2 U_{y} \frac{\partial U_{y}}{\partial x}+2 U_{z} \frac{\partial U_{z}}{\partial x} \\
=-\frac{1}{\sqrt{1-U^{2}}}\left(U_{x} \frac{\partial U_{x}}{\partial x} U_{x}+U_{y} \frac{\partial U_{y}}{\partial x} U_{x}+U_{z} \frac{\partial U_{z}}{\partial x} U_{x}\right) \\
=-\frac{1}{\sqrt{1-U^{2}}}\left(U_{x} \frac{\partial U_{x}}{\partial t}+U_{y} \frac{\partial U_{y}}{\partial t}+U_{z} \frac{\partial U_{z}}{\partial t}\right)=-\frac{1}{\sqrt{1-U^{2}}}\left(\mathbf{U} \cdot \frac{\partial \mathbf{U}}{\partial t}\right) \\
m\left(\mathbf{U} \cdot \boldsymbol{\nabla}\left(\sqrt{1-U^{2}}\right)\right)=-\frac{3 m}{\sqrt{1-U^{2}}}(\mathbf{U} \cdot \mathbf{a})
\end{gathered}
$$

We obtain

$$
\begin{aligned}
\frac{4 m}{\left(\sqrt{1-U^{2}}\right)^{3}}(\mathbf{U} \cdot \mathbf{a})-\frac{3 m}{\sqrt{1-U^{2}}}(\mathbf{U} \cdot \mathbf{a}) & \\
& =q(\mathbf{U} \cdot \mathbf{E})+q(\mathbf{U} \cdot(\mathbf{U} \times \operatorname{rot} \mathbf{A}))+q(\mathbf{U} \cdot((\mathbf{U} \boldsymbol{\nabla}) \mathbf{A}))+q(\mathbf{U} \cdot(\mathbf{A} \times \operatorname{rot} \mathbf{U}))
\end{aligned}
$$

Simplify the left part

$$
\begin{gathered}
\frac{4 m}{\left(\sqrt{1-U^{2}}\right)^{3}}(\mathbf{U} \cdot \mathbf{a})-\frac{3 m}{\sqrt{1-U^{2}}}(\mathbf{U} \cdot \mathbf{a})= \\
\frac{m}{\sqrt{1-U^{2}}}(\mathbf{U} \cdot \mathbf{a})\left(\frac{4}{1-U^{2}}-3\right)=\frac{m}{\sqrt{1-U^{2}}}(\mathbf{U} \cdot \mathbf{a})\left(\frac{4-3+3 U^{2}}{1-U^{2}}\right) \\
=\frac{m}{\sqrt{1-U^{2}}}(\mathbf{U} \cdot \mathbf{a})\left(\frac{1+3 U^{2}}{1-U^{2}}\right)
\end{gathered}
$$

$(\mathbf{U} \cdot \mathbf{a}) \frac{m\left(1+3 U^{2}\right)}{\left(\sqrt{1-U^{2}}\right)^{3}}=q(\mathbf{U} \cdot \mathbf{E})+q(\mathbf{U} \cdot(\mathbf{U} \times \operatorname{rot} \mathbf{A}))+q(\mathbf{U} \cdot((\mathbf{U} \boldsymbol{\nabla}) \mathbf{A}))+q(\mathbf{U} \cdot(\mathbf{A} \times \operatorname{rot} \mathbf{U}))$ 


$$
\begin{gathered}
(\mathbf{U} \cdot \mathbf{a})=\frac{q}{m} \frac{\left(\sqrt{1-U^{2}}\right)^{3}}{1+3 U^{2}}((\mathbf{U} \cdot \mathbf{E})+(\mathbf{U} \cdot(\mathbf{U} \times \operatorname{rot} \mathbf{A}))+(\mathbf{U} \cdot((\mathbf{U} \nabla) \mathbf{A}))+(\mathbf{U} \cdot(\mathbf{A} \times \operatorname{rot} \mathbf{U}))) \\
\frac{d \varepsilon}{d t}=\frac{4 q}{1+3 U^{2}}((\mathbf{U} \cdot \mathbf{E})+(\mathbf{U} \cdot(\mathbf{U} \times \operatorname{rot} \mathbf{A}))+(\mathbf{U} \cdot((\mathbf{U} \mathbf{\nabla}) \mathbf{A}))+(\mathbf{U} \cdot(\mathbf{A} \times \operatorname{rot} \mathbf{U}))) \\
\frac{d \mathbf{P}}{d t}=\frac{4 m \mathbf{a}}{\sqrt{1-U^{2}}}+\frac{4 \mathbf{U} q}{1+3 U^{2}}((\mathbf{U} \cdot \mathbf{E})+(\mathbf{U} \cdot(\mathbf{U} \times \operatorname{rot} \mathbf{A}))+(\mathbf{U} \cdot((\mathbf{U V}) \mathbf{A}))+(\mathbf{U} \cdot(\mathbf{A} \times \operatorname{rot} \mathbf{U}))) \\
\frac{\partial}{\partial t}\left(\frac{m U^{2}}{2}\right)=\frac{\left(\sqrt{1-U^{2}}\right)^{3}}{1+3 U^{2}} q((\mathbf{U} \cdot \mathbf{E})+(\mathbf{U} \cdot(\mathbf{U} \times \operatorname{rot} \mathbf{A}))+(\mathbf{U} \cdot((\mathbf{U V}) \mathbf{A})) \\
+(\mathbf{U} \cdot(\mathbf{A} \times \operatorname{rot} \mathbf{U})))
\end{gathered}
$$

In the presence of an electric field only, we have

$$
\begin{gathered}
\frac{d \mathcal{E}}{d t}=\frac{4 q}{\left(1+3 U^{2}\right)}(\mathbf{U} \cdot \mathbf{E}) \\
\frac{\partial}{\partial t}\left(\frac{m U^{2}}{2}\right)=\frac{\left(\sqrt{1-U^{2}}\right)^{3}}{1+3 U^{2}} q(\mathbf{U} \cdot \mathbf{E}) \\
\frac{d \mathbf{P}}{d t}=\frac{4 m \mathbf{a}}{\sqrt{1-U^{2}}}+\frac{4 \mathbf{U}}{\left(1+3 U^{2}\right)} q(\mathbf{U} \cdot \mathbf{E})
\end{gathered}
$$

The force depends on both velocity and acceleration, the electric field, which also depends on velocity and acceleration, can be defined by the formula

$$
\frac{1}{q} \frac{d \mathbf{P}}{d t}=\frac{m}{q} \frac{4 \mathbf{a}}{\sqrt{1-U^{2}}}+\frac{4 \mathbf{U}}{\left(1+3 U^{2}\right)}(\mathbf{U} \cdot \mathbf{E})
$$

After transformations, which will be described later in the article, of the right part of the Lagrange equation at a nonzero vector potential, it takes the form

$$
\begin{gathered}
\frac{d \mathcal{E}}{d t}=\frac{4 q}{\left(1+3 U^{2}\right)}\left((\mathbf{U} \cdot \mathbf{E})+2(\mathbf{U} \cdot \mathbf{a})\left(\mathbf{A} \cdot \frac{1}{\mathbf{U}}\right)-3(\mathbf{A} \cdot \mathbf{a})\right) \\
\frac{d \mathbf{P}}{d t}=\frac{4 m \mathbf{a}}{\sqrt{1-U^{2}}}+\frac{4 \mathbf{U} q}{1+3 U^{2}}\left((\mathbf{U} \cdot \mathbf{E})+2(\mathbf{U} \cdot \mathbf{a})\left(\mathbf{A} \cdot \frac{1}{\mathbf{U}}\right)-3(\mathbf{A} \cdot \mathbf{a})\right) \\
\frac{\partial}{\partial t}\left(\frac{m U^{2}}{2}\right)=\frac{\left(\sqrt{1-U^{2}}\right)^{3}}{1+3 U^{2}} q\left((\mathbf{U} \cdot \mathbf{E})+2(\mathbf{U} \cdot \mathbf{a})\left(\mathbf{A} \cdot \frac{1}{\mathbf{U}}\right)-3(\mathbf{A} \cdot \mathbf{a})\right)
\end{gathered}
$$

Consider the case where the field is created by a stationary charge and so the vector potential is zero. Then the force acting on the moving charge is

$$
\frac{d \mathbf{P}}{d t}=-m \boldsymbol{\nabla}\left(\sqrt{1-U^{2}}\right)-q \boldsymbol{\nabla} \varphi=q \mathbf{E}_{\mathrm{s}}-m \boldsymbol{\nabla}\left(\sqrt{1-U^{2}}\right)
$$

Let us compare this expression with the previously obtained expression

$$
\frac{d \mathbf{P}}{d t}=q \mathbf{E}_{\mathbf{U}}-\gamma^{2} q\left(\widehat{\mathbf{R}} \times\left(\mathbf{E}_{\mathbf{U}} \times \mathbf{a}\right)\right)=\gamma q \mathbf{E}_{\mathbf{s}}-q \Gamma \mathbf{U}\left(\mathbf{U} \cdot \mathbf{E}_{\mathbf{s}}\right)-\gamma^{2} q\left(\widehat{\mathbf{R}} \times\left(\mathbf{E}_{\mathbf{U}} \times \mathbf{a}\right)\right)
$$

It turns out that

$$
\begin{gathered}
q \mathbf{E}_{\mathrm{s}}-m \boldsymbol{\nabla}\left(\sqrt{1-U^{2}}\right)=\gamma q \mathbf{E}_{\mathrm{s}}-q \Gamma \mathbf{U}\left(\mathbf{U} \cdot \mathbf{E}_{\mathrm{s}}\right)-\gamma^{2} q\left(\widehat{\mathbf{R}} \times\left(\mathbf{E}_{\mathbf{U}} \times \mathbf{a}\right)\right) \\
\mathbf{E}_{\mathbf{s}}=Q \frac{\mathbf{r}}{r^{3}}
\end{gathered}
$$

Both the left and right parts depend on velocity and on $\mathbf{r}$. All the terms are forces acting on the particle.

The total energy is equal to the sum of kinetic and potential energy and for it we have 
$\frac{d}{d t}\left(\frac{m}{\sqrt{1-U^{2}}}+\frac{q Q}{r}\right)=q\left(\mathbf{U} \cdot \mathbf{E}_{\mathrm{s}}\right)-m\left(\mathbf{U} \cdot \boldsymbol{\nabla}\left(\sqrt{1-U^{2}}\right)\right)-\frac{q Q}{r^{2}} \frac{d r}{d t}=-m\left(\mathbf{U} \cdot \boldsymbol{\nabla}\left(\sqrt{1-U^{2}}\right)\right)$ Let us compare with

$$
\frac{d}{d t}\left(\frac{m}{\sqrt{1-U^{2}}}+\frac{q Q}{r}\right)=-q \gamma^{2}\left(\mathbf{U} \cdot\left(\widehat{\mathbf{R}} \times\left(\mathbf{E}_{\mathbf{U}} \times \mathbf{a}\right)\right)\right)
$$

The following equation is obtained

$$
-m\left(\mathbf{U} \cdot \boldsymbol{\nabla}\left(\sqrt{1-U^{2}}\right)\right)=-q \gamma^{2}\left(\mathbf{U} \cdot\left(\widehat{\mathbf{R}} \times\left(\mathbf{E}_{\mathbf{U}} \times \mathbf{a}\right)\right)\right)
$$

Let's express the left part through acceleration

$$
\begin{gathered}
\boldsymbol{\nabla}\left(\sqrt{1-U^{2}}\right)=\left(\frac{\partial\left(\sqrt{1-U^{2}}\right)}{\partial x}, \frac{\partial\left(\sqrt{1-U^{2}}\right)}{\partial y}, \frac{\partial\left(\sqrt{1-U^{2}}\right)}{\partial z}\right) \\
\frac{\partial\left(\sqrt{1-U^{2}}\right)}{\partial x}=-\frac{1 / 2}{\sqrt{1-U^{2}}} \frac{\partial U^{2}}{\partial x}=-\frac{1}{\sqrt{1-U^{2}}}\left(U_{x} \frac{\partial U_{x}}{\partial x}+U_{y} \frac{\partial U_{y}}{\partial x}+U_{z} \frac{\partial U_{z}}{\partial x}\right) \\
\frac{\partial U^{2}}{\partial x}=\frac{\partial}{\partial x}\left(U_{x}^{2}+U_{y}^{2}+U_{z}^{2}\right)=2 U_{x} \frac{\partial U_{x}}{\partial x}+2 U_{y} \frac{\partial U_{y}}{\partial x}+2 U_{z} \frac{\partial U_{z}}{\partial x} \\
\frac{\partial U_{x}}{\partial x}=\frac{\partial U_{x}}{\partial t} \frac{\partial t}{\partial x}=\frac{a_{x}}{U_{x}} \\
\frac{\partial U_{y}}{\partial x}=\frac{\partial U_{y}}{\partial t} \frac{\partial t}{\partial x}=\frac{a_{y}}{U_{x}} \\
\frac{\partial U_{z}}{\partial x}=\frac{\partial U_{z}}{\partial t} \frac{\partial t}{\partial x}=\frac{a_{z}}{U_{x}} \\
-m \boldsymbol{\nabla}\left(\sqrt{1-U^{2}}\right)=m\left(\frac{(\mathbf{U} \cdot \mathbf{a})}{\partial x} \frac{1}{\sqrt{1-U^{2}}}, \frac{(\mathbf{U} \cdot \mathbf{a})}{\sqrt{1-U^{2}}} \frac{1}{U_{y}}, \frac{(\mathbf{U} \cdot \mathbf{a})}{\sqrt{1-U^{2}}} \frac{1}{U_{z}}\right)=m \frac{(\mathbf{U} \cdot \mathbf{a})}{\sqrt{1-U^{2}}}\left(\frac{1}{U_{x}}, \frac{1}{U_{y}}, \frac{1}{U_{z}}\right) \\
=m \frac{(\mathbf{U} \cdot \mathbf{a})}{\sqrt{1-U^{2}} \frac{1}{\mathbf{U}}}\left(U_{x} \frac{a_{x}}{U_{x}}+U_{y} \frac{a_{y}}{U_{x}}+U_{z} \frac{a_{z}}{U_{x}}\right)=-\frac{(\mathbf{U} \cdot \mathbf{a})}{\sqrt{1-U^{2}}} \frac{1}{U_{x}}
\end{gathered}
$$

where

$$
\begin{gathered}
\frac{1}{\mathbf{U}} \equiv\left(\frac{1}{U_{x}}, \frac{1}{U_{y}}, \frac{1}{U_{z}}\right) \\
-m\left(\mathbf{U} \cdot \boldsymbol{\nabla}\left(\sqrt{1-U^{2}}\right)\right)=m \frac{(\mathbf{U} \cdot \mathbf{a})}{\sqrt{1-U^{2}}}\left(\frac{1}{U_{x}}, \frac{1}{U_{y}}, \frac{1}{U_{z}}\right)\left(U_{x}, U_{y}, U_{z}\right)^{T} \\
=m \frac{(\mathbf{U} \cdot \mathbf{a})}{\sqrt{1-U^{2}}}\left(\frac{U_{x}}{U_{x}}+\frac{U_{y}}{U_{y}}+\frac{U_{z}}{U_{z}}\right)=3 m \frac{(\mathbf{U} \cdot \mathbf{a})}{\sqrt{1-U^{2}}}
\end{gathered}
$$

It turns out that

$$
3 m \frac{(\mathbf{U} \cdot \mathbf{a})}{\sqrt{1-U^{2}}}=-q \gamma^{2}\left(\mathbf{U} \cdot\left(\widehat{\mathbf{R}} \times\left(\mathbf{E}_{\mathbf{U}} \times \mathbf{a}\right)\right)\right)
$$

This equation cannot be considered accurate, because the expression on the left takes into account all radiation losses, both electromagnetic and gravitational, and the formula on the right reflects only the losses on electromagnetic radiation.

Let's look again at the general formula

$$
\frac{d \mathbf{P}}{d t}=-m \boldsymbol{\nabla}\left(\sqrt{1-U^{2}}\right)-q \frac{\partial \mathbf{A}}{\partial t}-q \boldsymbol{\nabla} \varphi+q(\mathbf{U} \times \operatorname{rot} \mathbf{A})+q(\mathbf{U} \boldsymbol{\nabla}) \mathbf{A}+q(\mathbf{A} \times \operatorname{rot} \mathbf{U})
$$


Express the terms in the right-hand side through velocity and acceleration

$$
\begin{aligned}
& \operatorname{rot} \mathbf{U}=(\boldsymbol{\nabla} \times \mathbf{U})=\left[\begin{array}{ccc}
\mathbf{i} & \mathbf{j} & \mathbf{k} \\
\frac{\partial}{\partial x} & \frac{\partial}{\partial y} & \frac{\partial}{\partial z} \\
U_{x} & U_{y} & U_{z}
\end{array}\right]=\mathbf{i}\left(\frac{\partial U_{z}}{\partial y}-\frac{\partial U_{y}}{\partial z}\right)-\mathbf{j}\left(\frac{\partial U_{z}}{\partial x}-\frac{\partial U_{x}}{\partial z}\right)+\mathbf{k}\left(\frac{\partial U_{y}}{\partial x}-\frac{\partial U_{x}}{\partial y}\right) \\
& =\mathbf{i}\left(\frac{\partial U_{z}}{\partial t} \frac{\partial t}{\partial y}-\frac{\partial U_{y}}{\partial t} \frac{\partial t}{\partial z}\right)-\mathbf{j}\left(\frac{\partial U_{z}}{\partial t} \frac{\partial t}{\partial x}-\frac{\partial U_{x}}{\partial t} \frac{\partial t}{\partial z}\right)+\mathbf{k}\left(\frac{\partial U_{y}}{\partial t} \frac{\partial t}{\partial x}-\frac{\partial U_{x}}{\partial t} \frac{\partial t}{\partial y}\right) \\
& =\mathbf{i}\left(a_{z} \frac{1}{U_{y}}-a_{y} \frac{1}{U_{z}}\right)-\mathbf{j}\left(a_{z} \frac{1}{U_{x}}-a_{x} \frac{1}{U_{z}}\right)+\mathbf{k}\left(a_{y} \frac{1}{U_{x}}-a_{x} \frac{1}{U_{y}}\right) \\
& =\left(\mathbf{a} \times\left(\frac{1}{U_{x}}, \frac{1}{U_{y}}, \frac{1}{U_{z}}\right)\right)=\left(\mathbf{a} \times \frac{1}{\mathbf{U}}\right) \\
& (\mathbf{A} \times \operatorname{rot} \mathbf{U})=\left(\mathbf{A} \times\left(\mathbf{a} \times \frac{1}{\mathbf{U}}\right)\right)=\mathbf{a}\left(\mathbf{A} \cdot \frac{1}{\mathbf{U}}\right)-\frac{1}{\mathbf{U}}(\mathbf{A} \cdot \mathbf{a}) \\
& (\mathbf{U} \cdot(\mathbf{A} \times \operatorname{rot} \mathbf{U}))=(\mathbf{U} \cdot \mathbf{a})\left(\mathbf{A} \cdot \frac{1}{\mathbf{U}}\right)-\left(\mathbf{U} \cdot \frac{1}{\mathbf{U}}\right)(\mathbf{A} \cdot \mathbf{a})=(\mathbf{U} \cdot \mathbf{a})\left(\mathbf{A} \cdot \frac{1}{\mathbf{U}}\right)-3(\mathbf{A} \cdot \mathbf{a}) \\
& (\mathbf{U \nabla}) \mathbf{A}=\left[\begin{array}{ccc}
\frac{\partial U_{x}}{\partial x} & \frac{\partial U_{x}}{\partial y} & \frac{\partial U_{x}}{\partial z} \\
\frac{\partial U_{y}}{\partial x} & \frac{\partial U_{y}}{\partial y} & \frac{\partial U_{y}}{\partial z} \\
\frac{\partial U_{z}}{\partial x} & \frac{\partial U_{z}}{\partial y} & \frac{\partial U_{z}}{\partial z}
\end{array}\right]\left[\begin{array}{l}
A_{x} \\
A_{y} \\
A_{z}
\end{array}\right]=\left[\begin{array}{l}
A_{x} \frac{\partial U_{x}}{\partial x}+A_{y} \frac{\partial U_{x}}{\partial y}+A_{z} \frac{\partial U_{x}}{\partial z} \\
A_{x} \frac{\partial U_{y}}{\partial x}+A_{y} \frac{\partial U_{y}}{\partial y}+A_{z} \frac{\partial U_{y}}{\partial z} \\
A_{x} \frac{\partial U_{z}}{\partial x}+A_{y} \frac{\partial U_{z}}{\partial y}+A_{z} \frac{\partial U_{z}}{\partial z}
\end{array}\right]= \\
& =\left[\begin{array}{l}
A_{x} \frac{a_{x}}{U_{x}}+A_{y} \frac{a_{x}}{U_{y}}+A_{z} \frac{a_{x}}{U_{z}} \\
A_{x} \frac{a_{y}}{U_{x}}+A_{y} \frac{a_{y}}{U_{y}}+A_{z} \frac{a_{y}}{U_{z}} \\
A_{x} \frac{a_{z}}{U_{x}}+A_{y} \frac{a_{z}}{U_{y}}+A_{z} \frac{a_{z}}{U_{z}}
\end{array}\right]=\left(A_{x} \frac{1}{U_{x}}+A_{y} \frac{1}{U_{y}}+A_{z} \frac{1}{U_{z}}\right)\left[\begin{array}{l}
a_{x} \\
a_{y} \\
a_{z}
\end{array}\right]=\left(\mathbf{A} \cdot \frac{1}{\mathbf{U}}\right) \mathbf{a} \\
& (\mathbf{U} \cdot((\mathbf{U \nabla}) \mathbf{A}))=(\mathbf{U} \cdot \mathbf{a})\left(\mathbf{A} \cdot \frac{1}{\mathbf{U}}\right)
\end{aligned}
$$

the result is

$\frac{d \mathbf{P}}{d t}=m \frac{(\mathbf{U} \cdot \mathbf{a})}{\sqrt{1-U^{2}}} \frac{1}{\mathbf{U}}-q \frac{\partial \mathbf{A}}{\partial t}-q \boldsymbol{\nabla} \varphi+q(\mathbf{U} \times \operatorname{rot} \mathbf{A})+q \mathbf{a}\left(\mathbf{A} \cdot \frac{1}{\mathbf{U}}\right)-q \frac{1}{\mathbf{U}}(\mathbf{A} \cdot \mathbf{a})+q \mathbf{a}\left(\mathbf{A} \cdot \frac{1}{\mathbf{U}}\right)$

For the derivative of kinetic energy

$$
\begin{gathered}
\frac{d \mathcal{E}}{d t}=3 m \frac{(\mathbf{U} \cdot \mathbf{a})}{\sqrt{1-U^{2}}}-q\left(\mathbf{U} \cdot \frac{\partial \mathbf{A}}{\partial t}\right)-q(\mathbf{U} \cdot \boldsymbol{\nabla} \varphi)+q(\mathbf{U} \cdot(\mathbf{U} \times \operatorname{rot} \mathbf{A}))+q(\mathbf{U} \cdot \mathbf{a})\left(\mathbf{A} \cdot \frac{1}{\mathbf{U}}\right) \\
-3 q(\mathbf{A} \cdot \mathbf{a})+q(\mathbf{U} \cdot \mathbf{a})\left(\mathbf{A} \cdot \frac{1}{\mathbf{U}}\right)
\end{gathered}
$$

For the derivative of total energy 


$$
\begin{aligned}
\frac{d}{d t}\left(\frac{m}{\sqrt{1-U^{2}}}+\right. & q \varphi)= \\
& =q \frac{d \varphi}{d t}+3 m \frac{(\mathbf{U} \cdot \mathbf{a})}{\sqrt{1-U^{2}}}-q\left(\mathbf{U} \cdot \frac{\partial \mathbf{A}}{\partial t}\right)-q(\mathbf{U} \cdot \boldsymbol{\nabla} \varphi)+q(\mathbf{U} \cdot(\mathbf{U} \times \operatorname{rot} \mathbf{A})) \\
& +q(\mathbf{U} \cdot \mathbf{a})\left(\mathbf{A} \cdot \frac{1}{\mathbf{U}}\right)-3 q(\mathbf{A} \cdot \mathbf{a})+q(\mathbf{U} \cdot \mathbf{a})\left(\mathbf{A} \cdot \frac{1}{\mathbf{U}}\right)
\end{aligned}
$$

Taking into account the found expressions, we obtain the previously given relations

$$
\begin{gathered}
(\mathbf{U} \cdot \mathbf{a})=\frac{q}{m} \frac{\left(\sqrt{1-U^{2}}\right)^{3}}{1+3 U^{2}}\left((\mathbf{U} \cdot \mathbf{E})+2(\mathbf{U} \cdot \mathbf{a})\left(\mathbf{A} \cdot \frac{1}{\mathbf{U}}\right)-3(\mathbf{A} \cdot \mathbf{a})\right) \\
\frac{d \varepsilon}{d t}=\frac{4 q}{\left(1+3 U^{2}\right)}\left((\mathbf{U} \cdot \mathbf{E})+2(\mathbf{U} \cdot \mathbf{a})\left(\mathbf{A} \cdot \frac{1}{\mathbf{U}}\right)-3(\mathbf{A} \cdot \mathbf{a})\right) \\
\frac{d \mathbf{P}}{d t}=\frac{4 m \mathbf{a}}{\sqrt{1-U^{2}}}+\frac{4 q \mathbf{U}}{1+3 U^{2}}\left((\mathbf{U} \cdot \mathbf{E})+2(\mathbf{U} \cdot \mathbf{a})\left(\mathbf{A} \cdot \frac{1}{\mathbf{U}}\right)-3(\mathbf{A} \cdot \mathbf{a})\right) \\
\frac{\partial}{\partial t}\left(\frac{m U^{2}}{2}\right)=\frac{\left(\sqrt{1-U^{2}}\right)^{3}}{1+3 U^{2}} q\left((\mathbf{U} \cdot \mathbf{E})+2(\mathbf{U} \cdot \mathbf{a})\left(\mathbf{A} \cdot \frac{1}{\mathbf{U}}\right)-3(\mathbf{A} \cdot \mathbf{a})\right) \\
\frac{4 m}{\left(\sqrt{1-U^{2}}\right)^{3}}(\mathbf{a}+(\mathbf{U} \times(\mathbf{U} \times \mathbf{a}))) \\
=\frac{4 m \mathbf{a}}{\sqrt{1-U^{2}}}+\frac{4 \mathbf{U} q}{1+3 U^{2}}\left((\mathbf{U} \cdot \mathbf{E})+2(\mathbf{U} \cdot \mathbf{a})\left(\mathbf{A} \cdot \frac{1}{\mathbf{U}}\right)-3(\mathbf{A} \cdot \mathbf{a})\right)
\end{gathered}
$$

Consider the motion of a charged particle at zero vector potential in the electric field $\mathbf{E}$, measured at a fixed observation point

$$
\frac{d \mathbf{P}}{d t}=-m \boldsymbol{\nabla}\left(\sqrt{1-U^{2}}\right)-q \boldsymbol{\nabla} \varphi=m \frac{(\mathbf{U} \cdot \mathbf{a})}{\sqrt{1-U^{2}}} \frac{1}{\mathbf{U}}+q \mathbf{E}
$$

It is clear from this that the force acting on a charge in an electric field is not reduced to the value of $q \mathbf{E}$. The derivative of kinetic energy is

$$
\frac{d \varepsilon}{d t}=3 m \frac{(\mathbf{U} \cdot \mathbf{a})}{\sqrt{1-U^{2}}}+q(\mathbf{U} \cdot \mathbf{E})
$$

We can find the expression for the full time derivative of velocity from the relation

$$
\begin{gathered}
\frac{d \mathbf{P}}{d t}=\mathbf{U} \frac{d}{d t}\left(\frac{m}{\sqrt{1-U^{2}}}\right)+\frac{m}{\sqrt{1-U^{2}}} \frac{d \mathbf{U}}{d t} \\
\frac{d \mathbf{U}}{d t}=\sqrt{1-U^{2}}\left(\frac{(\mathbf{U} \cdot \mathbf{a})}{\sqrt{1-U^{2}}} \frac{1}{\mathbf{U}}-3 \mathbf{U} \frac{(\mathbf{U} \cdot \mathbf{a})}{\sqrt{1-U^{2}}}+\frac{q}{m} \mathbf{E}-\frac{q}{m} \mathbf{U}(\mathbf{U} \cdot \mathbf{E})\right)
\end{gathered}
$$

On the other hand, we can express it through acceleration

$$
\frac{d \mathbf{U}}{d t}=\frac{\partial \mathbf{U}}{\partial t}+(\mathbf{U} \boldsymbol{\nabla}) \mathbf{U}=\frac{\partial \mathbf{U}}{\partial t}+\left(\mathbf{U} \cdot \frac{1}{\mathbf{U}}\right) \mathbf{a}=\mathbf{a}+3 \mathbf{a}=4 \mathbf{a}
$$

Let us compare two ways of calculating the force acting on an initially resting charge in a constant homogeneous electric field. The field, force, velocity, and acceleration all point in the same direction, so scalar and vector product are easy to calculate. In the first method we use the ratios

$$
\frac{d \mathbf{P}}{d t}=\frac{4 m}{\left(\sqrt{1-U^{2}}\right)^{3}}(\mathbf{a}+(\mathbf{U} \times(\mathbf{U} \times \mathbf{a})))
$$




$$
\frac{d \mathbf{P}}{d t}=m \frac{(\mathbf{U} \cdot \mathbf{a})}{\sqrt{1-U^{2}}} \frac{1}{\mathbf{U}}+q \mathbf{E}
$$

By equating the right-hand sides, we obtain

$$
\begin{gathered}
\frac{4 m}{\left(\sqrt{1-U^{2}}\right)^{3}}(\mathbf{a}+(\mathbf{U} \times(\mathbf{U} \times \mathbf{a})))=m \frac{(\mathbf{U} \cdot \mathbf{a})}{\sqrt{1-U^{2}}} \frac{1}{\mathbf{U}}+q \mathbf{E} \\
\frac{4 m a}{\left(\sqrt{1-U^{2}}\right)^{3}}=m \frac{U a}{\sqrt{1-U^{2}}} \frac{1}{U}+q E \\
4 a \frac{1}{\left(\sqrt{1-U^{2}}\right)^{3}}=\frac{a}{\sqrt{1-U^{2}}}+\frac{q}{m} E \\
4 a=a\left(1-U^{2}\right)+\left(\sqrt{1-U^{2}}\right)^{3} \frac{q}{m} E \\
4 a=a\left(1-U^{2}\right)+\frac{q}{m} E\left(\sqrt{1-U^{2}}\right)^{3} \\
a\left(3+U^{2}\right)=\frac{q}{m} E\left(\sqrt{1-U^{2}}\right)^{3} \\
a=\frac{q}{m} E \frac{\left(\sqrt{1-U^{2}}\right)^{3}}{\left(3+U^{2}\right)}
\end{gathered}
$$

Let's substitute the acceleration into the expression for the force

$$
\frac{d \mathbf{P}}{d t}=\frac{4 m a}{\left(\sqrt{1-U^{2}}\right)^{3}}=\frac{4 m}{\left(\sqrt{1-U^{2}}\right)^{3}} \frac{q}{m} E \frac{\left(\sqrt{1-U^{2}}\right)^{3}}{\left(3+U^{2}\right)}=\frac{4}{3} q E \frac{1}{\left(1+\frac{1}{3} U^{2}\right)}
$$

In the second method we use the ratio

$$
\begin{gathered}
\frac{d \mathcal{E}}{d t}=3 m \frac{(\mathbf{U} \cdot \mathbf{a})}{\sqrt{1-U^{2}}}+q(\mathbf{U} \cdot \mathbf{E}) \\
\frac{d \mathbf{P}}{d t}=\frac{d(\mathbf{U} \mathcal{E})}{d t}=\mathbf{U} \frac{d \mathcal{E}}{d t}+\varepsilon \frac{d \mathbf{U}}{d t} \\
\frac{d \mathbf{U}}{d t}=\frac{1}{\varepsilon}\left(\frac{d \mathbf{P}}{d t}-\mathbf{U} \frac{d \mathcal{E}}{d t}\right) \\
\frac{d \mathbf{U}}{d t}=\frac{\sqrt{1-U^{2}}}{m}\left(m \frac{(\mathbf{U} \cdot \mathbf{a})}{\sqrt{1-U^{2}}} \frac{1}{\mathbf{U}}+q \mathbf{E}-\mathbf{U} \frac{d \mathcal{E}}{d t}\right)
\end{gathered}
$$

We obtain

$$
\begin{gathered}
\frac{d \mathbf{U}}{d t}=4 \frac{\partial \mathbf{U}}{\partial t}=4 \mathbf{a}=\frac{\sqrt{1-U^{2}}}{m}\left(m \frac{(\mathbf{U} \cdot \mathbf{a})}{\sqrt{1-U^{2}}} \frac{1}{\mathbf{U}}+q \mathbf{E}-\mathbf{U}\left(3 m \frac{(\mathbf{U} \cdot \mathbf{a})}{\sqrt{1-U^{2}}}+q(\mathbf{U} \cdot \mathbf{E})\right)\right) \\
\left.4 a=\frac{\sqrt{1-U^{2}}}{m}\left(m \frac{U a}{\sqrt{1-U^{2}}} \frac{1}{U}+q E-3 m \frac{U U a}{\sqrt{1-U^{2}}}-q E U U\right)\right) \\
\left.4 a=\sqrt{1-U^{2}}\left(\frac{a}{\sqrt{1-U^{2}}}+\frac{q}{m} E-3 \frac{U^{2} a}{\sqrt{1-U^{2}}}-\frac{q}{m} E U^{2}\right)\right) \\
4 a=\sqrt{1-U^{2}}\left(\frac{a\left(1-3 U^{2}\right)}{\sqrt{1-U^{2}}}+\frac{q}{m} E\left(1-U^{2}\right)\right) \\
4 a=a\left(1-3 U^{2}\right)+\frac{q}{m} E\left(\sqrt{1-U^{2}}\right)^{3}
\end{gathered}
$$




$$
\begin{gathered}
a\left(3+3 U^{2}\right)=\frac{q}{m} E\left(\sqrt{1-U^{2}}\right)^{3} \\
a=\frac{q}{m} E \frac{\left(\sqrt{1-U^{2}}\right)^{3}}{\left(3+3 U^{2}\right)} \\
\frac{d \mathbf{P}}{d t}=\frac{a m}{\sqrt{1-U^{2}}}+q E=q E+\frac{m}{\sqrt{1-U^{2}}} \frac{q}{m} E \frac{\left(\sqrt{1-U^{2}}\right)^{3}}{3+3 U^{2}}=q E+q E \frac{1-U^{2}}{3+3 U^{2}} \\
=q E \frac{3+3 U^{2}+1-U^{2}}{3+3 U^{2}}=q E \frac{4+2 U^{2}}{3+3 U^{2}}=\frac{4}{3} q E \frac{1+\frac{1}{2} U^{2}}{1+U^{2}}
\end{gathered}
$$

At zero velocity in both methods of calculation the force is equal to $4 / 3 q E$, and at a speed equal to the speed of light it is equal to $q E$.

If we take as the value of the electric field the force of its action divided by the value of the charge, we can see from this example that the field in a moving observation point depends on its speed, although in this particular case the formula does not include acceleration.

It turns out that, taking into account all the terms of the Lagrange equation, that is, without ignoring the dependence of velocity on coordinates, the same field acts on the particle with $4 / 3$ time the force, that is, in a sense, its mass is effectively $4 / 3$ times less, then, substituting this smaller mass in the formula for electromagnetic energy from [2] (Chapter 28, Paragraph 3, page 311 )

$$
U_{\text {эл }}=4 / 3 m_{\text {эм }} c^{2}
$$

We get the correct relationship between electromagnetic energy and mass.

It would seem that there is a logical contradiction in the fact that the force acting on a particle with zero velocity is not equal to $q \mathbf{E}$, because by $\mathbf{E}$ we just mean the field at a stationary point of observation. But this refers to a really stationary point that has not only no velocity but also no acceleration, e.g. a rigidly fixed point. We know that the field depends on the acceleration, but a free stationary particle in the electric field has it and therefore the field has a larger value $4 / 3 q \mathbf{E}$. So it is natural that there is a larger force acting on the particle than on the fixed particle. As the speed increases, the field decreases, and when it reaches the speed of light, when the particle stops accelerating, the force again becomes equal to $q \mathbf{E}$.

For the case of a constant and homogeneous electric field, we were able to find the value of acceleration $a$, but in the general case we do not know a simple formula for the vector $\mathbf{a}$.

Consider motion only in a constant and homogeneous magnetic field, when the scalar potential is zero. We know from experience that velocity and acceleration are orthogonal in a constant and homogeneous magnetic field. We obtain

$$
\frac{d}{d t}\left(\frac{m}{\sqrt{1-U^{2}}}+q \varphi\right)=\frac{d}{d t}\left(\frac{m}{\sqrt{1-U^{2}}}\right)=-3 q(\mathbf{A} \cdot \mathbf{a})
$$

Thus, when a charge moves in a magnetic field, its energy does change due to radiation. This fact is known from the motion of particles in a synchrotron, in which it is necessary to feed them with energy to keep their trajectories close to circles. And since the trajectories deviate from circles, the scalar product of velocity and acceleration is different from zero and it is necessary to consider the contribution to radiation and those terms in which this scalar product enters.

It is also interesting to consider the case when both scalar and vector potentials are constant and homogeneous, but not equal to zero, then there is no magnetic and electric field 


$$
\begin{gathered}
\frac{d \mathbf{P}}{d t}=m \frac{(\mathbf{U} \cdot \mathbf{a})}{\sqrt{1-U^{2}}} \frac{1}{\mathbf{U}}-q \frac{\partial \mathbf{A}}{\partial t}-q \boldsymbol{\nabla} \varphi+q(\mathbf{U} \times \operatorname{rot} \mathbf{A})+q \mathbf{a}\left(\mathbf{A} \cdot \frac{1}{\mathbf{U}}\right)-q \frac{1}{\mathbf{U}}(\mathbf{A} \cdot \mathbf{a})+q \mathbf{a}\left(\mathbf{A} \cdot \frac{1}{\mathbf{U}}\right) \\
=m \frac{(\mathbf{U} \cdot \mathbf{a})}{\sqrt{1-U^{2}}} \frac{1}{\mathbf{U}}+2 q \mathbf{a}\left(\mathbf{A} \cdot \frac{1}{\mathbf{U}}\right)-q \frac{1}{\mathbf{U}}(\mathbf{A} \cdot \mathbf{a})
\end{gathered}
$$

The right part contains an expression for the force acting on the particle in the presence of vector potential only, without any field.

The application of Lorentz transformations to the velocity four-vector and the momentum four-vector is valid only when the velocity of the particle does not change. In this case, the transformed momentum includes the new velocity, which coincides with the velocity obtained from the velocity addition formula. But the formula of addition of velocities is valid only if both velocities included in the formula do not change in time and space. If in some frame of reference there is a particle moving with a certain speed and acceleration, and we want to find the speed of this particle in another frame of reference which moves uniformly with respect to the first, we cannot do it, because the formula of addition of speeds is applicable only if both speeds are constant, and if the particle moves accelerated, we do not yet know the formula of addition of speeds for it, because its speed in the new frame depends on the acceleration of the particle. In this case we cannot calculate the momentum of the particle in the other frame of reference, we can only measure it.

Consider two inertial systems. Then the quantities with the dashed line refer to the moving system. The $x$-axes of both systems are parallel to each other and their relative velocities $\mathbf{v}$. Consider the interval of time and space between two close sequential events. For each of these events, we can write down the Lorentz transformations and find their difference, then we obtain

$$
d t^{\prime}=\frac{d t-v d x}{\sqrt{1-v^{2}}} \quad d x^{\prime}=\frac{d x-v d t}{\sqrt{1-v^{2}}}
$$

Let the events consist of a moving object passing through these points with speed $\mathbf{u}$ and acceleration $\mathbf{a}$ in a stationary system, then for the projections of all quantities on the $x$ and $x^{\prime}$ axes we obtain

We can write

$$
d x=u d t+a(d t)^{2} \quad d x^{\prime}=u^{\prime} d t^{\prime}+a^{\prime}\left(d t^{\prime}\right)^{2}
$$

$$
\begin{gathered}
u^{\prime} d t^{\prime}+a^{\prime}\left(d t^{\prime}\right)^{2}=\frac{u d t+a(d t)^{2}-v d t}{\sqrt{1-v^{2}}} \\
u^{\prime}+a^{\prime} d t^{\prime}=\frac{u \frac{d t}{d t^{\prime}}+a d t \frac{d t}{d t^{\prime}}-v \frac{d t}{d t^{\prime}}}{\sqrt{1-v^{2}}} \\
\frac{d t}{d t^{\prime}}=\frac{d t \sqrt{1-v^{2}}}{d t-v d x}=\frac{\sqrt{1-v^{2}}}{1-v \frac{d x}{d t}}=\frac{\sqrt{1-v^{2}}}{1-v(u+a d t)} \\
u^{\prime}+a^{\prime} d t^{\prime}=\frac{u+a d t-v}{1-v(u+a d t)}
\end{gathered}
$$

If both accelerations are zero, we get the usual formula for the projection of velocities on the $x$ and $x^{\prime}$ axes

$$
u^{\prime}=\frac{u-v}{1-v u}
$$

In the general case of nonzero accelerations, however, we do not obtain such a simple formula, and we have yet neglected higher order derivatives. In some cases, acceleration can be 
neglected, but in the equation of motion of a charged particle in the electromagnetic field acceleration must be taken into account since a force acts on the particle which changes its momentum, i.e. it gives acceleration, and this force itself depends on acceleration. Therefore, when approaching the equation of motion from the position of covariance requirement, it is necessary to take acceleration into account in transformations of quantities included in the equation. Where the velocity itself enters, e.g., $\frac{1}{\sqrt{1-U^{2}}}$, acceleration, in view of the smallness of $d t$, can be neglected in comparison to velocity, but where the velocity derivative is included, e.g., $m \frac{(\mathbf{U} \cdot \mathbf{a})}{\sqrt{1-U^{2}}} \frac{1}{\mathbf{U}}$, acceleration $d t$ cannot be neglected, since it is included without the infinitely small multiplier $d t$.

Thus, the concept of covariance in the sense of the Lorentz transformation is incorrect to apply to the equation of motion of a charged particle in the electromagnetic field, since the Lorentz transformation assumes uniformity of all motions involved, while the equation of motion deals with forces and accelerations, which excludes uniformity of motion. That's when all parts of the equation of motion are transformed according to the two-parameter group of transformations that account for acceleration, then it will be true covariance.

If velocity is not constant, then the equation of motion cannot be called covariant in the context of Lorentz transformations. In another frame of reference, the equation would be the same, but with velocities and accelerations that cannot be calculated using the Lorentz transformation, even if the systems are mutually inertial.

The energy-momentum four-vector is a four-vector vector only as long as it does not change. If it changes, it ceases to be a four-vector. Accordingly, it is not very correct to consider the equation of motion in the tensor representation.

So far, the difficulties in dealing with the equation of motion of a charged particle in the electromagnetic field are often circumvented by stating that velocity does not depend on coordinates and therefore discarding the terms that depend on its change in space.

The Lagrange function and the Lorentz transformation for the scalar potential are similar; in fact, the Lagrange function involves a transformation of the scalar potential from a stationary observation point to an observation point moving with speed $\mathbf{U}$.

$$
\begin{gathered}
L=-m \sqrt{1-U^{2}}-q \hat{\varphi}+q(\mathbf{U} \cdot \widehat{\mathbf{A}}) \\
\varphi=\frac{1}{\sqrt{1-U^{2}}}(\hat{\varphi}-(\mathbf{U} \cdot \widehat{\mathbf{A}}))
\end{gathered}
$$

Although the Lorentz transformations have the disadvantages noted above for the case of non-uniform motion of the charge, this does not apply to potentials, since it follows from the equations for potentials that they do not depend on the acceleration of the charges creating them, but only on their speed and location. That is, the transformations for the potentials will not change, when the correct transformations for the fields and momentum of the particle will be found, taking into account the acceleration. Therefore, we can be sure of absolute correctness of transformation for scalar potential and for Lagrange function, on the one hand, and on the other hand we are sure of correctness of solutions of equations for potentials (18) and (19).

Having found these solutions $\widehat{\mathbf{A}}$ and $\hat{\varphi}$, we can substitute them into the equation of motion

$$
\frac{d \mathbf{P}}{d t}=m \frac{(\mathbf{U} \cdot \mathbf{a})}{\sqrt{1-U^{2}}} \frac{1}{\mathbf{U}}-q \frac{\partial \widehat{\mathbf{A}}}{\partial t}-q \boldsymbol{\nabla} \hat{\varphi}+q(\mathbf{U} \times \operatorname{rot} \widehat{\mathbf{A}})+q \mathbf{a}\left(\widehat{\mathbf{A}} \cdot \frac{1}{\mathbf{U}}\right)-q \frac{1}{\mathbf{U}}(\widehat{\mathbf{A}} \cdot \mathbf{a})+q \mathbf{a}\left(\widehat{\mathbf{A}} \cdot \frac{1}{\mathbf{U}}\right)
$$




$$
\begin{aligned}
& \frac{d \mathcal{E}}{d t}=3 m \frac{(\mathbf{U} \cdot \mathbf{a})}{\sqrt{1-U^{2}}}-q\left(\mathbf{U} \cdot \frac{\partial \widehat{\mathbf{A}}}{\partial t}\right)-q(\mathbf{U} \cdot \boldsymbol{\nabla} \hat{\varphi})+q(\mathbf{U} \cdot(\mathbf{U} \times \operatorname{rot} \widehat{\mathbf{A}}))+q(\mathbf{U} \cdot \mathbf{a})\left(\widehat{\mathbf{A}} \cdot \frac{1}{\mathbf{U}}\right)-3 q(\widehat{\mathbf{A}} \cdot \mathbf{a})+q(\mathbf{U} \cdot \mathbf{a})\left(\widehat{\mathbf{A}} \cdot \frac{1}{\mathbf{U}}\right) \\
& \frac{d}{d t}\left(\frac{m}{\sqrt{1-U^{2}}}+q \hat{\varphi}\right)= \\
&=q \frac{d \hat{\varphi}}{d t}+3 m \frac{(\mathbf{U} \cdot \mathbf{a})}{\sqrt{1-U^{2}}}-q\left(\mathbf{U} \cdot \frac{\partial \widehat{\mathbf{A}}}{\partial t}\right)-q(\mathbf{U} \cdot \boldsymbol{\nabla} \hat{\varphi})+q(\mathbf{U} \cdot(\mathbf{U} \times \operatorname{rot} \widehat{\mathbf{A}})) \\
&+q(\mathbf{U} \cdot \mathbf{a})\left(\widehat{\mathbf{A}} \cdot \frac{1}{\mathbf{U}}\right)-3 q(\widehat{\mathbf{A}} \cdot \mathbf{a})+q(\mathbf{U} \cdot \mathbf{a})\left(\widehat{\mathbf{A}} \cdot \frac{1}{\mathbf{U}}\right)
\end{aligned}
$$

Perhaps we have unnecessary problems using the concept of a field in the context of the equation of motion. Fields are useful in solving other problems, but they are inconvenient in the equation of motion. For any configuration of moving charges, we know their potentials and thus can find a solution to our exact, without simplifying assumptions, equation of motion of a particle, at least numerically.

This approach does not deny the possibility of subsequently returning to the use of the concept of field and forces by substituting into the right part of the equation of motion at once the correct expressions for the fields in non-uniform motion when the necessary group transformation has been found. Then we will not substitute the expressions $\hat{\varphi}$ and $\widehat{A}$ in the equation of motion, we will substitute only the correct expression for the force in the form of the electric field $\mathbf{E}$ for the moving observation point multiplied by the magnitude of the charge in the right-hand side of the equation for the derivative of momentum.

In the case of several arbitrarily moving charged particles, a similar equation is true for each of them. The system of these interdependent equations can also be solved by iterative numerical methods. The calculations are simplified by the fact that for each of the particles there are analytic expressions for $-q \frac{\partial \widehat{\mathbf{A}}}{\partial t}-q \boldsymbol{\nabla} \hat{\varphi}$ and $\operatorname{rot} \widehat{\mathbf{A}}$, since these are the electric and magnetic fields of an arbitrarily moving charge at a stationary observation point. The terms $q \mathbf{a}\left(\widehat{\mathbf{A}} \cdot \frac{1}{\mathbf{U}}\right)$ and $q \frac{1}{\mathbf{U}}(\widehat{\mathbf{A}} \cdot \mathbf{a})$ are also known because $\mathrm{A}^{\wedge}$ are the Lienard-Wiechert potentials of an arbitrarily moving charge. Although the expressions for the fields are valid for the stationary point of observation only, but when summed with the other terms in the right-hand side, they determine the force acting already on an arbitrarily moving charge.

Let us clarify the logic of our reasoning. The starting point for us is the equation for potentials at a given distribution of charges and currents. However, there is an auxiliary way to describe potentials - for simplicity we can speak not about potentials, but about fields corresponding to them in a fixed point of observation. This is just a way to characterize potentials. There is an equation of motion, in it one must substitute the potentials at a fixed point of observation, and the velocity and acceleration of a particle will be taken into account by the equation of motion itself. And there is another way to take into account the motion - it is again to start from potentials or corresponding fields for the stationary observation point and recalculate them already in the fields for the moving observation point, and then multiply the electric field for the moving point by the charge and get the force acting on it.

If we talk about analytical solutions, at small deviations of motion from uniformity we can neglect all the terms depending on acceleration, as it is often done. But, in contrast to the 
generally accepted approach, we can use the formula for the electric field in uniform motion. Let's return to the differences between the two approaches to writing the equation of motion. One consists in writing the Lagrange equation, neglecting all the terms that depend on acceleration, and expressing the remaining terms containing the scalar and vector potential through the electric and magnetic field for the stationary observation point, so that only the Lorentz force remains in the equation. In the second approach, the force acting on the moving charge in the electromagnetic field is immediately written on the right-hand side, usually taking the Lorentz force again as such a force. It seems that in both cases the result is the same. But the second approach is wrong in principle, because it is not the Lorentz force in its standard interpretation that acts on a moving charge, because the field at the moving point of observation does not coincide with the field at the stationary point of observation. We know the form of this field in uniform motion, in non-uniform motion we do not know. The first approach gives the correct solution if we do not neglect the terms that depend on acceleration. Without knowing the field for non-uniform motion, we can substitute at least the field for uniform motion into the expression for the force; it does not account for acceleration, but, unlike when using the Lorentz force, it at least accounts for velocity.

$$
\begin{gathered}
\mathbf{E}_{\mathbf{U}}=\gamma(\mathbf{E}+(\mathbf{U} \times \mathbf{B}))-\Gamma \mathbf{U}(\mathbf{U} \cdot \mathbf{E}) \\
\frac{d \mathbf{P}}{d t}=q \gamma(\mathbf{E}+(\mathbf{U} \times \mathbf{B}))-q \Gamma \mathbf{U}(\mathbf{U} \cdot \mathbf{E})=q \gamma \mathbf{E}+q \gamma(\mathbf{U} \times \mathbf{B})-q \Gamma \mathbf{U}(\mathbf{U} \cdot \mathbf{E}) \\
\frac{d \varepsilon}{d t}=\left(\mathbf{U} \cdot(q \gamma \mathbf{E}+q \gamma(\mathbf{U} \times \mathbf{B})-q \Gamma \mathbf{U}(\mathbf{U} \cdot \mathbf{E}))=(\mathbf{U} \cdot \mathbf{E}) q \gamma-q \Gamma U^{2}(\mathbf{U} \cdot \mathbf{E})\right. \\
=(\mathbf{U} \cdot \mathbf{E})\left(q \gamma-q \Gamma U^{2}\right)=q(\mathbf{U} \cdot \mathbf{E})\left(\gamma-\Gamma U^{2}\right)=q(\mathbf{U} \cdot \mathbf{E})
\end{gathered}
$$

One can find the expression for the full derivative of the velocity of the particle from the relation

$$
\begin{gathered}
\frac{d \mathbf{P}}{d t}=\mathbf{U} \frac{d \varepsilon}{d t}+\frac{m}{\sqrt{1-U^{2}}} \frac{d \mathbf{U}}{d t}=q \mathbf{U}(\mathbf{U} \cdot \mathbf{E})+\gamma m \frac{d \mathbf{U}}{d t} \\
\gamma m \frac{d \mathbf{U}}{d t}=q \gamma \mathbf{E}+q \gamma(\mathbf{U} \times \mathbf{B})-q \Gamma \mathbf{U}(\mathbf{U} \cdot \mathbf{E})-q \mathbf{U}(\mathbf{U} \cdot \mathbf{E}) \\
\frac{d \mathbf{U}}{d t}=\frac{q}{m}(\mathbf{E}-(\Gamma+1) \mathbf{U}(\mathbf{U} \cdot \mathbf{E})+(\mathbf{U} \times \mathbf{B}))
\end{gathered}
$$

Let us compare the result with the conventional approach

$$
\begin{gathered}
\frac{d \mathbf{P}}{d t}=q \mathbf{E}+q(\mathbf{U} \times \mathbf{B}) \\
\frac{d \mathcal{E}}{d t}=(\mathbf{U} \cdot(q \mathbf{E}+q(\mathbf{U} \times \mathbf{B}))=q(\mathbf{U} \cdot \mathbf{E}) \\
\frac{d \mathbf{P}}{d t}=\mathbf{U} \frac{d \varepsilon}{d t}+\frac{m}{\sqrt{1-U^{2}}} \frac{d \mathbf{U}}{d t}=q \mathbf{U}(\mathbf{U} \cdot \mathbf{E})+\gamma m \frac{d \mathbf{U}}{d t} \\
\gamma m \frac{d \mathbf{U}}{d t}=q \mathbf{E}+q(\mathbf{U} \times \mathbf{B})-q \mathbf{U}(\mathbf{U} \cdot \mathbf{E}) \\
\frac{d \mathbf{U}}{d t}=\frac{q}{m} \frac{1}{\gamma}(\mathbf{E}-\mathbf{U}(\mathbf{U} \cdot \mathbf{E})+(\mathbf{U} \times \mathbf{B}))
\end{gathered}
$$


The difference in the results is the more significant, the closer is the speed of the particle to the speed of light. But there is a difference only in the value of acceleration, the derivative of the kinetic energy in both cases is the same and does not take into account the radiation of the accelerated moving charge.

There is another option to obtain the equation of motion in the constant velocity approximation - substitute in it the expressions from the equations of electrodynamics as we know them at the moment, that is just for the uniform motion of the observation point

$$
\begin{gathered}
\operatorname{rot} \widehat{\mathbf{A}}=\widehat{\mathbf{B}}=\gamma(\mathbf{B}+(\mathbf{v} \times \mathbf{E}))-\Gamma \mathbf{v}(\mathbf{v} \cdot \mathbf{B}) \\
-\boldsymbol{\nabla} \hat{\varphi}-\frac{\partial \widehat{\mathbf{A}}}{\partial t}=\widehat{\mathbf{E}}=\gamma(\mathbf{E}-(\mathbf{v} \times \mathbf{B}))-\Gamma \mathbf{v}(\mathbf{v} \cdot \mathbf{E})
\end{gathered}
$$

by putting $\mathbf{v}=\mathbf{U}$, and neglecting all the terms depending on acceleration we obtain

$$
\begin{aligned}
\frac{d \mathbf{P}}{d t}=m \frac{(\mathbf{U} \cdot \mathbf{a})}{\sqrt{1-U^{2}}} \frac{1}{\mathbf{U}}-q \frac{\partial \widehat{\mathbf{A}}}{\partial t}-q \boldsymbol{\nabla} \widehat{\varphi}+q(\mathbf{U} \times \operatorname{rot} \widehat{\mathbf{A}})+q \mathbf{a}\left(\widehat{\mathbf{A}} \cdot \frac{1}{\mathbf{U}}\right)-q \frac{1}{\mathbf{U}}(\widehat{\mathbf{A}} \cdot \mathbf{a}) \\
\quad+q \mathbf{a}\left(\widehat{\mathbf{A}} \cdot \frac{1}{\mathbf{U}}\right)=-q \frac{\partial \widehat{\mathbf{A}}}{\partial t}-q \boldsymbol{\nabla} \widehat{\varphi}+q(\mathbf{U} \times r o t \widehat{\mathbf{A}}) \\
=q \gamma(\mathbf{E}-(\mathbf{v} \times \mathbf{B}))-q \Gamma \mathbf{v}(\mathbf{v} \cdot \mathbf{E})+q(\mathbf{U} \times(\gamma(\mathbf{B}+(\mathbf{v} \times \mathbf{E}))-\Gamma \mathbf{v}(\mathbf{v} \cdot \mathbf{B}))) \\
=q \gamma \mathbf{E}-q \gamma(\mathbf{v} \times \mathbf{B})-q \Gamma \mathbf{v}(\mathbf{v} \cdot \mathbf{E})+q \gamma(\mathbf{U} \times \mathbf{B})+\gamma(\mathbf{U} \times(\mathbf{v} \times \mathbf{E})) \\
=q \gamma \mathbf{E}-q \Gamma \mathbf{v}(\mathbf{v} \cdot \mathbf{E})+q \gamma(\mathbf{U} \times(\mathbf{v} \times \mathbf{E})) \\
=q \gamma \mathbf{E}-q \Gamma \mathbf{U}(\mathbf{U} \cdot \mathbf{E})+q \gamma(\mathbf{U}(\mathbf{U} \cdot \mathbf{E}))-q \gamma(\mathbf{E}(\mathbf{U} \times \mathbf{U})) \\
=q \gamma \mathbf{E}-q \gamma \mathbf{E} U^{2}-q \Gamma \mathbf{U}(\mathbf{U} \cdot \mathbf{E})+q \gamma \mathbf{U}(\mathbf{U} \cdot \mathbf{E}) \\
=q \frac{1}{\gamma} \mathbf{E}+q \gamma \mathbf{U}(\mathbf{U} \cdot \mathbf{E})-q \Gamma \mathbf{U}(\mathbf{U} \cdot \mathbf{E})
\end{aligned}
$$

The magnetic field has disappeared, which is understandable, because we assume that motion is uniform, and this is only possible when the magnetic field is zero, which causes acceleration. At low velocity, as it should be, only the electric field remains for the stationary observation point

$$
\frac{d \mathbf{P}}{d t}=q \mathbf{E}
$$

It appears that the expression

$$
\frac{d \mathbf{P}}{d t}=q \frac{1}{\gamma} \mathbf{E}+q \gamma \mathbf{U}(\mathbf{U} \cdot \mathbf{E})-q \Gamma \mathbf{U}(\mathbf{U} \cdot \mathbf{E})
$$

is a better approximation than the standard approach

$$
\frac{d \mathbf{P}}{d t}=q \mathbf{E}+q(\mathbf{U} \times \mathbf{B})
$$

or substituting into the right hand side of the field of a uniformly moving charge

$$
\frac{d \mathbf{P}}{d t}=q \gamma \mathbf{E}+q \gamma(\mathbf{U} \times \mathbf{B})-q \Gamma \mathbf{U}(\mathbf{U} \cdot \mathbf{E})
$$

because it is obtained directly from the equation of motion and the equation for potentials.

The electromagnetic field tensor defined according to [2] (Chapter 26, paragraph 3) should now be written as follows to give the equations of electrodynamics a covariant form 


$$
\left(\hat{F}_{\mu \nu}\right)=\left(\begin{array}{cccc}
0 & \hat{B}_{z} & -\hat{B}_{y} & -\hat{E}_{x} \\
-\hat{B}_{z} & 0 & \hat{B}_{x} & -\hat{E}_{y} \\
\hat{B}_{y} & -\hat{B}_{x} & 0 & -\widehat{E}_{z} \\
\hat{E}_{x} & \hat{E}_{y} & \hat{E}_{z} & 0
\end{array}\right)
$$

In this case (30) and (32) can be written in covariant form as equality of the tensor divergence to the four-vector current with minus sign. And (31) and (33) can be written in covariant form as four homogeneous equations for the tensor components.

In the tensor, the velocity components of the observation point are functions of coordinates and time in the reference frame used, so in the general case they cannot all be made zero by moving to another reference frame, unless the velocity field is constant and homogeneous. If one tries to work with an observation point with zero velocity, the velocity in it will still become nonzero when one moves to another frame of reference. As noted above, if we use the electromagnetic field tensor to write the equation of motion of a charged particle in the covariant form, it will be incorrect even in this form, which takes into account the speed of the observation point. The motion is still uniform, and the very notion of the equation of motion implies that the velocity is not constant under the action of the field. Since so far we can substitute in the electromagnetic field tensor only the transformations for a uniformly moving observation point, this tensor is not suitable for describing arbitrary motion of the observation point. Therefore, in particular, it is incorrect to use this tensor when dealing with the equation of motion of a charged particle in the electromagnetic field; such an approach, for example, does not allow us to consider radiation during the accelerated motion of the charge.

Consider the law of conservation of energy. The work performed by the electric field per unit time in a unit volume is equal to

given the identity

$$
(\widehat{\mathbf{E}} \cdot \hat{\mathbf{j}})=\varepsilon_{0} c^{2}(\widehat{\mathbf{E}} \cdot \operatorname{rot} \widehat{\mathbf{B}})-\varepsilon_{0}\left(\widehat{\mathbf{E}} \cdot \frac{\partial}{\partial t} \widehat{\mathbf{E}}\right)
$$

and the equations of electrodynamics, we obtain

$$
\operatorname{div}(\widehat{\mathbf{E}} \times \widehat{\mathbf{B}})=\widehat{\mathbf{B}} \cdot \operatorname{rot} \widehat{\mathbf{E}}-\widehat{\mathbf{E}} \cdot \operatorname{rot} \widehat{\mathbf{B}}
$$

$$
\begin{aligned}
(\widehat{\mathbf{E}} \cdot \hat{\mathbf{j}})=\varepsilon_{0} c^{2}(\widehat{\mathbf{B}} \cdot \operatorname{rot} \hat{\mathbf{E}})-\varepsilon_{0} c^{2} \operatorname{div}(\widehat{\mathbf{E}} \times \widehat{\mathbf{B}})-\varepsilon_{0}\left(\widehat{\mathbf{E}} \cdot \frac{\partial}{\partial t} \hat{\mathbf{E}}\right) & \\
= & -\varepsilon_{0} c^{2}\left(\widehat{\mathbf{B}} \cdot \frac{\partial}{\partial t} \widehat{\mathbf{B}}\right)-\varepsilon_{0} c^{2} \operatorname{div}(\widehat{\mathbf{E}} \times \widehat{\mathbf{B}})-\varepsilon_{0}\left(\widehat{\mathbf{E}} \cdot \frac{\partial}{\partial t} \widehat{\mathbf{E}}\right)= \\
& =-\varepsilon_{0}\left(c^{2} \operatorname{div}(\widehat{\mathbf{E}} \times \widehat{\mathbf{B}})+c^{2}\left(\widehat{\mathbf{B}} \cdot \frac{\partial}{\partial t} \widehat{\mathbf{B}}\right)+\left(\widehat{\mathbf{E}} \cdot \frac{\partial}{\partial t} \widehat{\mathbf{E}}\right)\right)
\end{aligned}
$$

We define the total field energy density as

then we get

$$
\begin{gathered}
U=\frac{\varepsilon_{0}}{2}\left(c^{2}(\widehat{\mathbf{B}} \cdot \widehat{\mathbf{B}})+(\widehat{\mathbf{E}} \cdot \widehat{\mathbf{E}})\right) \\
\frac{\partial}{\partial t} U=\varepsilon_{0} c^{2}\left(\widehat{\mathbf{B}} \cdot \frac{\partial}{\partial t} \widehat{\mathbf{B}}\right)+\left(\widehat{\mathbf{E}} \cdot \frac{\partial}{\partial t} \widehat{\mathbf{E}}\right)
\end{gathered}
$$

Let's define the Poynting vector

$$
(\widehat{\mathbf{E}} \cdot \hat{\mathbf{j}})=-\varepsilon_{0} c^{2} \operatorname{div}(\widehat{\mathbf{E}} \times \widehat{\mathbf{B}})-\frac{\partial}{\partial t} U
$$




$$
\mathbf{S}=\varepsilon_{0} c^{2}(\widehat{\mathbf{E}} \times \widehat{\mathbf{B}})
$$

then

$$
\begin{gathered}
(\hat{\mathbf{E}} \cdot \hat{\mathbf{j}})=-\operatorname{div} \mathbf{S}-\frac{\partial}{\partial t} U \\
\frac{\partial}{\partial t} U+\operatorname{div} \mathbf{S}=-(\mathbf{E} \cdot \mathbf{j})
\end{gathered}
$$

Using the connection between the Poynting vector and the electromagnetic momentum density

we can write

$$
\mathbf{S}=c^{2} \mathbf{g}
$$

$$
\mathbf{g}=\varepsilon_{0}(\widehat{\mathbf{E}} \times \widehat{\mathbf{B}})
$$

Depending on the type of expressions for $\widehat{\mathbf{E}}$ and $\widehat{\mathbf{B}}$ - if the observation point moves uniformly or if it can be found arbitrarily, the definitions will be different.

\section{Alternator and generators}

Let us return to equation (6) for the electric field circulation.

$$
\oint_{L} \mathbf{E} \cdot d \mathbf{l}=\int_{S} \operatorname{rot} \mathbf{E} \cdot d \mathbf{s}=-\int_{S} \frac{\partial \mathbf{B}}{\partial t} \cdot d \mathbf{s}+\oint_{L}(\mathbf{v} \times \mathbf{B}) \cdot d \mathbf{l}-\frac{1}{c^{2}} \int_{S} \frac{\partial(\mathbf{v} \times \mathbf{E})}{\partial t} \cdot d \mathbf{s}
$$

If we close our eyes to the question of whether Stokes' theorem applies to a moving contour and a moving surface stretched over it, the last term in the right-hand side of the equation makes it possible to construct an alternating current generator without using a magnetic field. Let there be a constant electric field in the plane of the sheet, directed, for example, from right to left. In the plane of the sheet is a disk of non-conducting material, mounted on an axis perpendicular to the plane of the sheet. A conductive frame in the form of a sector of a circle, e.g. 60 degrees, connected to the contacts on the axis is fixed to the disk. The disk with the frame rotates in the plane of the sheet. Each point of imaginary flat surface stretched on the frame has a linear velocity, the vector of which changes from parallel to perpendicular to the field vector, respectively their vector product, directed along the normal to the frame plane, changes in module from zero to a maximum according to a sinusoidal law. By integrating the normal to surface component of the time derivative of the vector product at all points of the imaginary surface, we obtain the circulation of the electric field in the frame, which creates an EMF and a current when the circuit closes. The larger the opening angle of the frame, the larger the surface area, but at a certain angle the circulation will begin to decrease because of the phase difference of the derivative of the vector product on different sides of the frame. So at some angle of opening the EMF reaches the maximum. If you place two more such frames in a circle at equal distances, you get a semblance of a three-phase alternator, but because of integration over the surface the current will not be strictly sinusoidal. You can put several disks on the axis, connecting the circuits in parallel to sum the currents with the same phase.

When considering an alternator in a constant electric field, the question arises what forces will counteract the external force rotating the frame when current is generated in it. A similar question is what will happen if the frame is supplied with current from an external source, that is, will this device work as a motor. The answer, in our opinion, is as follows. The equations of electrodynamics are a law that is always satisfied. So if there is a combination of fields in which, 
according to the equations of electrodynamics, there must be some velocity, that velocity will appear, or rather efforts will be made to make it appear. This effort will create a counterforce to the external rotational force, as well as make the device work as a motor. More specifically, if there is current in the frame, there will be a magnetic field flow through the surface stretched over it, which will move and "pull" the frame with it. Thus, if an ordinary three-phase current is applied to a device with three frames evenly spaced on the disk, the disk will rotate and provide useful work.

Consider, taking into account the equations of electrodynamics, an experiment with two carts on rails, one of which has a permanent magnet attached and the other an ammeter, over the magnet a conductor is placed, connected through the rails to the ammeter. We have a closed circuit with the conductor. Let us compare two experiments: in the laboratory reference frame the cart with magnet rolls towards the second one, which stands, in this case the ammeter will fix the current, the second option - in the laboratory reference frame the cart with magnet stands, and the cart with ammeter rolls towards it, again the ammeter will fix the current. The third option - in the laboratory reference frame both carts roll with equal speed in one direction, then the ammeter will not fix the current.

In all three cases the first term in the right part of (6) is equal to zero, as the magnet is constant. If the cart with the magnet moves, then in the reference frame associated with the ammeter, the elements of the circuit have velocity in the magnetic field, a current is generated. If the cart with the ammeter moves and we are in its frame of reference, the cart with the magnet also moves and the circulation is different from zero, and the ammeter shows the presence of current. If both carts are moving at the same speed, then in the ammeter's frame of reference the conductor over the magnet is stationary, there is no circulation of the electric field and no current. That is, if we are all three times in the ammeter's frame of reference, it correctly shows that there is current when the magnet moves relative to the ammeter and no current when they are at rest relative to each other.

But we would like to be consistent and not to pass mentally from one frame of reference to another, but to consider these experiments only in one frame of reference, for example, the laboratory one, and to explain all results, relying on the equations of electrodynamics. The interaction of two charges was considered in the article earlier. A moving charge creates fields, but if the point where the second charge is located moves with the same speed, the fields of the first charge at this point are converted back to a Coulomb field. In the experiments under consideration, the mechanism of motion compensation is different. When a conductor moves in a magnetic field, an electric field is arising at its points, which is proportional to the value of magnetic induction and velocity. The combination of an electric and magnetic field creates a nonzero Poynting vector and a corresponding flux of energy. Due to the current in the circuit, a flux of energy and a nonzero Poynting vector, whose magnitude is proportional to the speed of the car with the magnet, is also generated in the stationary ammeter. When the ammeter moves, we must recalculate the fields from the stationary observation point to the moving one, and the recalculation formula must show that when the velocities of motion coincide, the Poynting vector becomes equal to zero, and if the velocities are not equal, then the formula of addition of velocities must work. The conversion to zero of the Poynting vector in the moving ammeter obviously occurs because the magnetic field at the moving observation point is zero after converting it from the field at the stationary point. 
But we do not know the exact magnitude of the magnetic and electric field in the conductor or in the ammeter, only their velocities. Therefore, it is not clear what values we should substitute in the recalculation formula, although we are sure that when the velocities are equal, the electric field in the ammeter will disappear. Thus, the mechanism for such precise compensation is not fully understood.

Consider the inverse in some sense of the problem with a cart with a magnet. Put a current source on the second cart instead of the ammeter and fix it. When there is current in the circuit, the Ampere force will push the cart with the magnet. If we attach the current source directly to the cart with the magnet, there will be no movement. Let's explain these results. The rule of thumb is this: When the power source is not attached to the cart with the magnet, the conductor in the magnetic field moves in the source frame of reference and the circulation of the electric field in the circuit is not zero, which generates a current that opposes the source current. If, on the other hand, the source is mounted on a cart with a magnet, the conductor in the magnetic field is stationary in the source frame of reference and the circulation is zero and there is no counteracting current. If in this case the cart were moving, it would move without resistance and the law of conservation of energy would be violated. That is, even in this case, it all depends on whether in the case of motion the nonzero circulation of the electric field in the reference frame of the current source is possible. If not, such motion is impossible

The same reasoning is true when considering the operation of disk unipolar generator and motor. If the ammeter is placed stationary in the laboratory frame of reference and the disk with the magnet rotates, the ammeter records the current because there is movement of the circuit part in the magnetic field in the frame of reference associated with it. If the disk is stationary and the platform with the ammeter rotates, again in the reference frame of the ammeter there is movement of the part of the circuit in the magnetic field and there is current. If the ammeter is fixed to the disk itself, all parts of the circuit in the ammeter reference frame are stationary and there is no current.

In a unipolar motor, if the current source is stationary in the laboratory reference frame, the disk rotates because, as indicated above in the case of cart motion, a counteracting current is generated in the disk. If the current source is fixed to the disk, the disk does not rotate because in the reference frame associated with the source, the circuit is stationary and there is no possibility of generating a counteracting current.

In equation (30) let's open parentheses and leave in the left part only the electric field rotor

$$
\begin{gathered}
\operatorname{rot}(\gamma \mathbf{E})=-\frac{\partial(\gamma \mathbf{B})}{\partial t}+\operatorname{rot}(\gamma(\mathbf{v} \times \mathbf{B}))-\frac{\partial(\gamma(\mathbf{v} \times \mathbf{E}))}{\partial t}+\frac{\partial(\Gamma \mathbf{v}(\mathbf{v} \cdot \mathbf{B}))}{\partial t}+\operatorname{rot}(\Gamma \mathbf{v}(\mathbf{v} \cdot \mathbf{E})) \\
\gamma=\frac{1}{\sqrt{1-v^{2}}} \quad \Gamma=\frac{\gamma-1}{v^{2}}
\end{gathered}
$$

The last two terms on the right-hand side give the possibility of generating an electric current in the longitudinal magnetic field and the longitudinal electric field. Let us write down only these two terms in integral form, omitting the previously considered

$$
\int_{S} \operatorname{rot}(\gamma \mathbf{E}) \cdot d \mathbf{s}=\int_{S} \operatorname{rot}(\Gamma \mathbf{v}(\mathbf{v} \cdot \mathbf{E})) \cdot d \mathbf{s}+\int_{S} \frac{\partial(\Gamma \mathbf{v}(\mathbf{v} \cdot \mathbf{B}))}{\partial t} \cdot d \mathbf{s}
$$




$$
\oint_{L} \gamma \mathbf{E} \cdot d \mathbf{l}=\oint_{L}(\Gamma \mathbf{v}(\mathbf{v} \cdot \mathbf{E})) \cdot d \mathbf{l}+\int_{S} \frac{\partial(\Gamma \mathbf{v}(\mathbf{v} \cdot \mathbf{B}))}{\partial t} \cdot d \mathbf{s}
$$

For the first summand in the right-hand side, assume that there is a disk rotating in the plane of the table, the constant electric field in the plane of the table is directed from right to left. The sign of the scalar product above and below the disk axis is opposite, so that the circulation of the electric field along the conducting contour passing along the edge of the disk is zero. If the field above the axis of the disk is directed in one direction and below the axis in the other direction, the circulation will be different from zero. At one point, the frame can be opened and brought out to the axis of the disk, where a direct current can be drawn. For more uniformity of current, the frame can be left closed and the current withdrawn with brushes. Several concentric frames can also be placed on the disk. Another way to use the longitudinal field is to leave the electric field homogeneous and use the disk with three frames described above. Then the EMF will be generated mainly on the edges of the frames, and the current will be alternating threephase.

For the second term, you can use a frame as in a conventional alternator in a constant magnetic field. If it is in the same constant homogeneous magnetic field as in an ordinary generator, the time derivative at the points on the surface stretched over the frame above and below the axis of rotation has a different sign, so that the integral of the derivative over the entire surface is zero. Therefore, for nonzero circulation it is necessary that above the axis of rotation the magnetic field is directed in one direction, and below the axis in the opposite direction. Then an alternating current can be drawn from the frame.

In both cases there is opposition to the force rotating the disk and the frame, and if you apply current to the frame, you get electric motors. The explanation for motor operation, as noted above, is that the equations of electrodynamics require the appearance of speed when creating a particular configuration of fields.

When creating a constant electric field parallel to the plane of the rotating disk, there may be technical difficulties due to the large diameter of the disk. It is much more convenient to place the disk parallel to the covers of a capacitor, also having the shape of a circle or a ring, and create a strong electric field. But then when rotating, the magnetic field will be directed radially and it makes no sense to place the frame on the disk. Therefore, let's place the frame on the disk rim so that the width of the frame fits between the capacitor's covers, and the length is about the same size. The ends of the frame will lead to the axis of the disk, where the linear velocity during rotation is minimal and it is easier to remove the current. With fast rotation, a strong magnetic field will pass through the area of the frame, but it will be constant and will not cause the electric field to circulate in the frame. Therefore, let's divide the capacitor into two semicircles and reverse the polarity of the capacitor on one of them. When the frame crosses the boundary of the capacitors, the magnetic field will change direction abruptly, so that the integral over the surface stretched over the frame from the derivative of the magnetic field will cause a strong short pulse of EMF in the frame. The capacitor can also be divided into four 90-degree sectors, but more importantly, many identical frames can be placed around the rim of the disk, and some can even be connected to each other if the pulses in them occur simultaneously. Thus, by applying sufficient torque to the disk, a frequent series of powerful current pulses can be obtained at the output. The pulse duration is determined by the length of the frame, which can reach up to half the length of the rim 


\section{Conclusion}

The equations of electrodynamics are presented, it is shown that plane and spherical electromagnetic waves are their solutions, while the spherical wave propagates only outward, which cannot be said about Maxwell's equations. The fields of uniformly moving charges are also solutions of the equations. The question of finding a universal form of the equations that allows a solution in the form of the field of an arbitrarily moving charge remains open. The question of the existence of a two-parameter group of transformations of electromagnetic fields along with the known one-parameter group has been posed. The phenomena derived from the equations, which make an additional contribution to the phase overrun in the Aharonov-Bohm effect are considered. The equation of motion of a charged particle in an electromagnetic field without simplifying approximations is considered. The principle of operation of an unconventional alternator in a constant electric field and a corresponding engine, as well as new types of generators of direct and impulse current, are described.

\section{References}

[1] Feynman Richard F., Leighton Robert B., Sands Matthew. Feynman Lectures in Physics. Issue. 5: Electricity and Magnetism. Librocom Book House. 2013. - 304 p.

[2] Feynman Richard F., Leighton Robert B., Sands Matthew. Feynman Lectures in Physics. Issue. 6: Electrodynamics. Librocom Book House. 2013. - 352 p.

[3] Sergey S. Stepanov. Relativistic World. Site synset.com.

[4] Landau L.D., Lifshits E.M. Theoretical Physics, Volume 2, Field Theory, 7th ed. - M. Nauka, 1988. - 512 p. 